\title{
CROMOSOMAS Y EVOLUCION EN ARACHIS (LEGUMINOSAE)
}

\author{
POI AVELIANO FERNÁNDEZ' y ANTONIO KRAPOVICKAS'
}

\section{Summary}

Forty-one species belonging to eight of the nine sections, of the genus Arachis were analyzed cytologically. In twenty-four aut of the forty-one species the karyotypes were analyzed in detail. All the species have one pair of satellited chromosomes, except one accession of $A$. valida, which had two pairs. Satellited chromosomes were classified in ten types. Sections Procumbentes, Erectoides, Extranervosae, Caulorrhizae, Rhizomatosae, Heteranthae and Trierectoides have only one or two types of satellited chromosomes, while sect. Arachis has six types. One pair of small chromosomes was found in sect. Arachis except in A. Batizocoi, A. ipaënsis, A. glandulifera, $A$. magna and $A$. benensis.

A. glandulifera (sect. Arachis) has the most asymmetrical karyotype, with six pairs of subtelocentric chromosomes, and A. guaranitica (sect. Trierectoides) has the most symmetrical karyotype. This support the hypotesis that sect. Trierectoides is the most ancient in the genus and that sect. Arachis is of a more recent origin.

Taking into account their karyotypes, external morphology and geographical distribution, $A$. duranensis and $A$. ipaënsis are probably the genome donors of $A$. hypogaea.

\section{Introducción}

El género Arachis L., originario de Sudamérica, cuenta con 69 especies descriptas (Krapovickas \& Gregory, 1994), la mayoría de las cuales son diploides $(2 n=2 x=20)$ y solamente cuatro tetraploides $(2 n=4 x=40)$. Entre estas últimas se encuentran dos especies de la sección Arachis, A. monticola (Krapovickas \& Rigoni, 1957) y A. hypogaea, (el maní cultivado) y dos de la sección Rhizomatosae, A. glabrata (Gregory, 1946) y A.pseudovillosa.

Husted $(1933,1936)$ fue el primero en estudiar la morfología de los cromosomas de $A$. hypogaea. Diferenció en el complemento dos pares, llamando cromosomas " $\mathrm{A}$ " a los del par más pequeño o diminuto y cromosomas " $\mathrm{B}$ " a los del par con constricción secundaria o cromosomas SAT. El par " $A$ " ha sido observado en $A$. correntina (sub A. villosa var. correntina)

'Miembros de la Carrera del Investigador Científico del CONICET

Instituto de Botánica del Nordeste, C.C. 209 - 3400 Corrientes
(Krapovickas \& Rigoni, 1951). Smartt (1964), al extender el estudio citológico a otras especies silvestres, observó la presencia del par " $\mathrm{A}$ " en varias especies de la sección Arachis y su ausencia en la sección Erectoides. A. Batizocoi es una especie de la sección Arachis en la cual no fue observado el par "A" (Krapovickas et al., 1974; Smartt et al., 1978; Stalker \& Dalmacio, 1981; Stalker et al., 1991a).

Stalker \& Dalmacio (1981) publicaron el cariotipo de 7 especies de la sección Arachis: $A$. Cardenasii, A. Diogoi (sub A. chacoensis), A. correntina, A. Batizocoi, A. duranensis (sub A. Spegazzinii), A. stenosperma y A. villosa. Singh \& Moss (1982) también dieron a conocer el cariotipo de las mismas especies y además, el del maní cultivado y el de $A$. monticola. Cai et al. (1987) estudiaron el cariotipo y bandeo-C de A. correntina, A. villosa, A. Cardenasii, $A$. Batizocoi, A. stenosperma, A. Rigonii, A. monticola y A. hypogaea.

En este trabajo se analiza la morfología de los cromosomas, especialmente del "A" y del cromosoma SAT de 40 especies silvestres que corresponden a 135 accesiones provenientes 
de Paraguay, Brasil, Bolivia y Argentina y 4 accesiones del maní cultivado. El estudio se realizó en todas las fases de la mitosis. El análisis mitótico en profase provee información útil para la relación filogenética en especies donde la condensación de los cromosomas se produce diferencialmente (Kurabayashi et al., 1962).

\section{Material y Métodos}

Se trabajó con semillas de las colecciones originales, la lista del material estudiado figura en la Tabla 1. Los ejemplares de herbario testigos están depositados en el herbario del Instituto de Botánica del Nordeste (CTES).

Tabla 1. Material estudiado y cromosomas.

\begin{tabular}{cc}
\hline Especie & 2n Crom. Coleccionista ${ }^{\circ}$ y procedencia \\
ASAT & \\
\hline
\end{tabular}

Sección Trierectoides Krapov.

\& W.C. Gregory

A. guaranitica Chodat \& $20-2 \quad$ GK 10568. Brasil, MS. Rio Amambai.

Hassl. Figs. 11A y 2

Sección Erectoides Krapov.

\& W.C. Gregory

A. Archeri Krapov. $20-2 \quad$ KSc 30159. Brasil, MS. 17 km S de Campo Grande.

\& W.C. Gregory

A. Benthamii Handro

20

$20-2$

$20-2$

$20-2$

A. gracilis Krapov.

\& W.C. Gregory

A. Hermannii Krapov.

\& W.C. Gregory

A. major Krapov.

\& W.C. Gregory

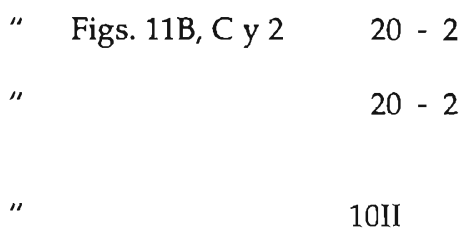

$20-2$

$20-2$

GKP 9841. Brasil, MS. 54 km N de Campo

Grande, Camino a Cuiabá.

HLKHe 551. Brasil, MS. 4 km E de J. Murtinho.

HLKHe 555a. Brasil, MS. 2 km E de Piraputanga.

HLKHe 559. Brasil, MS. Ruta a Nioaque, $40 \mathrm{~km} \mathrm{~S} \mathrm{de}$ Aquidauana.

GKP 9825. Brasil, MS. Aquidauana.

\footnotetext{
Abreviaturas: $\mathrm{Ah}=\mathrm{L} . \mathrm{Z}$. Ahumada, $\mathrm{Al}=\mathrm{D}$. Andrade-Lima, B=D.J. Banks, Bo=E.Bordas, Bu=A. Burkart, $\mathrm{Ca}=\mathrm{S}$. Cáceres, $\mathrm{C}=\mathrm{C}$.L. Cristóbal, $\mathrm{Cu}=\mathrm{C} . \mathrm{N}$. Cunha, $\mathrm{F}=\mathrm{A}$. Fernández, $\mathrm{Ge}=\mathrm{J} . \mathrm{M}$. González, $\mathrm{G}=$ W.C. Gregory, $\mathrm{Gr}=\mathrm{A}$. Gripp, $\mathrm{H}=\mathrm{R}$.O. Hammons, $\mathrm{He}=\mathrm{V}$. Hemsy, J=L. Jank, $\mathrm{K}=\mathrm{A}$. Krapovickas, $\mathrm{Kr}=\mathrm{A} . \mathrm{E}$. Kretschmer, $\mathrm{L}=$ W.R. Langford, $\mathrm{Mo}=\mathrm{L}$.A.Mroginski, $\mathrm{Oj}=\mathrm{H} . \mathrm{R}$. Ojeda, $\mathrm{P}=J . R$. Pietrarelli, Po=A. Pott, R=V.R. Rao, Ra=P.R. Rayman, Sc=A. Schinini, Sv=G.P. Silva, $S=C$.E. Simpson, $T=S . G$. Tressens, V=J.F. Valls, $V n=R . O$. Vanni, $\mathrm{Ve}=R . F . A$. Veiga, $W=W . L$. Werneck y $\mathrm{Zm}=\mathrm{C}$. Zamudio.
} 
20

20

20

$20-2$

$20-2$

$20-2$

$20-2$

$20-2$

$20-3$

A. paraguariensis ssp. capibarensis Krapov.

\& W. C. Gregory

" Figs. 11E, F y 2

"

“

A. paraguariensis Chodat

\& Hassl. ssp.

paraguariensis

$20-4$

$20-4$

$20-4$

" Fig. 2

$20-4$

" Fig. 2

$20-4$

GK 10575. Paraguay, Amambay. 54 km SW de P.J. Caballero.

GK 10580. Paraguay, Amambay. $70 \mathrm{~km}$ SW de P.J.

Caballero.

GK 10582. Paraguay, Amambay. Ñu Porá.

KHe 14444. Paraguay, Amambay. $45 \mathrm{~km}$ W de Pedro Juan Caballero.

KG 30016. Brasil, MS. Mun. Miranda, 12 km E de Miranda, camino a Aquidauana.

KGPSc 30128. Brasil, MS. 37 km E de Bela Vista, camino a Ponta Porã.

KSc 30151. Brasil, MS. 23 km E de Aquidauana camino a Campo Grande.

KSc 30153. Brasil, MS. 23 km E de Aquidauana camino a Campo Grande.

HLKHe 570. Brasil, MS.64 km E de Pto.Murtinho.

HLKHe 573. Brasil, MS. 108 km E de Pto.Murtinho.

KGPSc 30134. Brasil, MS. 44 km E de Pto.Murtinho, camino a Jardím.

KGPSc 30139. Brasil, MS. $82 \mathrm{~km}$ E de Pto.Murtinho, camino a Jardím.

KGPSc 30141. Brasil, MS. 83 km E de Pto.Murtinho, camino a Jardím.

GK 10562. Paraguay, Amambay. San Luis.

KC 11462. Paraguay, Cordillera. Tobatí.

KG 30013. Brasil, MS. Mun. Miranda, 16 km S de Guaicurus.

KG 30015. Brasil, MS. Mun. Miranda, Estancia Esmeralda, $45 \mathrm{~km}$ S de Guaicurus.

KGPSc 30109. Paraguay, Cordillera. 1 km N Ruta 2, camino de Ypacarai a S. Bernardino.

KGPSc 30110. Paraguay, Cordillera. 2 km de Tobatí, camino a Arroyos y Esteros.

KGPSc 30115. Paraguay, Amambay. 1 km N del río Aquidabán. 
$20-4 \quad$ KGPSc 30118. Paraguay, Amambay. $9 \mathrm{~km}$ SE de Bella Vista.

" Fig. 11D

$20-4$

$20-4$

$20-4$

A. paraguariensis aff. ssp. paraguariensis

A. stenophylla Krapov. \& W.C. Gregory. Figs. $11 G$ y 2

" Fig. 2

$20-4$

$20-4$

Sección Extranervosae Krapov. \& W.C. Gregory

A. Burchellii Krapov. \& W.C. Gregory

A. lutescens Krapov. et Rigoni

A. aff. prostrata Benth. Fig. $13 \mathrm{H}$

A. villosulicarpa Hoehne

$20-1$

$20-2$

Sección Heteranthae Krapov. \& W.C. Gregory

\section{A. Dardani Krapov.} \& W.C. Gregory

A. pusilla Benth.

A. sylvestris (A.Cheval.)

A.Cheval.

$20-10$

$20-10$

$20-10$

$20-10$

$20-10$
KGPSc 30124. Paraguay, Amambay. $20 \mathrm{~km}$ SE de Bella Vista.

KGPSc 30133. Brasil. MS. 71 km W de Jardím.

KGPSc 30142. Brasil, MS. 93 km E de Pto.Murtinho.

KC 11488. Paraguay, Ypacaraí, camino a San Bernardino.

HLKHe 572. Brasil, MS. 87 km E de Pto. Murtinho.

KGPSc 30126. Brasil, MS. 7 km E de Bela Vista, lomas arenosas del río Apa.

KGPSc 30136. Brasil, MS. 67 km E de Pto. Murtinho, camino a Jardím.

VKRSv 6637. Brasil, TO. $32 \mathrm{~km} \mathrm{~W} \mathrm{de} \mathrm{Pto.} \mathrm{Nacional.}$

VSGr 6332. Brasil, MT. $13 \mathrm{~km}$ WSW of Cuiaba.

VW 5913. Brasil, MG. Mun. Araguari. Faz.Piçarrão.

KHe 14445. Brasil, SP. Inst. Agron. Campinas.

VKSSv 8820. Brasil, MT. Mun. Vila Bela, Posto Indigena Mamainde $82 \mathrm{~km}$ SE de Vilhena.

VKVeSv 7039. Brasil, BA. Mun. Barreiras, Barreiras.

VRSv 10833. Brasil, BA. Mun. Barra, BR- 242, 11 km $W$ da ponte sobre rio Săo Francisco.

VKRSv 6547. Brasil, TO. 2 km S de Guarai, BR-153.

VKRSv 7071. Brasil, BA. BR-153, 200 m N da saída para Santa Rita de Cassia.

VRSv 10891. Brasil, BA. Pindobaçu.

VRSv 10980. Brasil, RN. Mun. Açu, 8,2 km W do rio Açu.

VRSv 11020. Brasil, CE. Tianguá. Unidade de

Pesquisa de lbiapaba/EPACE. 
Sección Caulorrhizae Krapov.

\& W.C. Gregory

A. Pintoi Krapov.

\& C.W. Gregory. Fig. 12B

$20-3$

$20-2$

Sección Procumbentes Krapov. \& W.C. Gregory

A. appressipila Krapov.

\& W.C. Gregory.

$20-9$

$20-9$

“ Fig. 12F

$20-9$

A. Kretschmeri Krapov. \& W.

$20-9$

C. Gregory. Figs. 12E y 3

$20-9$

$20-9$

A. lignosa (Chodat \& Hassl.)

Krapov. \& W.C. Gregory

Figs. $12 C$ y 3

A. matiensis Krapov.

\& W.C. Gregory

$20-9$

$20-9$

$20-9$

$20-9$

A. aff. matiensis Krapov.

\& W.C. Gregory

A. Rigonii Krapov.

$20-9$

\& W.C. Gregory . Fig. 12D

Sección Rhizomatosae Krapov.

\& W.C. Gregory

A. glabrata Benth.

var. glabrata

40

A. glabrata var.

$40-3$

Hagenbeckii (Harms ex

Kuntze) Herm.
GK 12787 Brasil, BA. IPEAL, Cruz das Almas.

VSW 6791. Brasil, GO. Formosa. $12 \mathrm{~km}$ NNW of Formosa, Faz. Jenipapo.

GKP 9993. Brasil, MS. Corumbá.

KG 30003. Brasil, MS. Corumbá, aeropuerto.

KG 30009. Brasil, MS. 30 km E de Corumbá, Fazenda Nova Campina.

K 34574. Brasil, MS. Campo Grande, cult. EMBRAPA.

KG 30007. Brasil, MS. 40 km de Corumbá, camino a Porto Manga.

$\mathrm{KrRa}$, Brasil, MS. cult. IRFL 2273, PI 446898, $36 \mathrm{~km}$ from Miranda on the road to Corumbá.

KCAh 14248. Paraguay, Concepción.

VSGr 6356. Brasil, MT. 49 km WNW of Cáceres, on road to Pto. Espiridião.

VSGr 6357. Brasil, MT. Porto Espiridião.

VSGr 6366. Brasil, MT. 66 km NW of Pto.Espiridião, on road to Pontes e Lacerda.

VSGr 6340. Brasil, MT. 26 km E of Cáceres.

GKP 10034. Bolivia, Santa Cruz de la Sierra.

GK 10596. Paraguay, Concepción, Horqueta.

F 385. Paraguay, Cordillera, Caacupé. 
A. pseudovillosa (Chodat \& 40 Hassl.) Krapov. \& W.C.

Gregory

\section{Sección Arachis}

A. Batizocoi Krapov. \& W.C. $20-5$ Gregory. Figs. 13A y 4

\section{A. benensis Krapov.}

\& W.C. Gregory

A. Cardenasii Krapov. \& W.

C. Gregory. Fig. 14A, B

$$
\text { "Fig. } 5
$$

$$
"
$$

A. correntina (Burkart) Krapov. et W. C. Gregory

Figs. $14 \mathrm{C}$ y 5

\section{A. Diogoi Hoehne}

" Fig. 7

A. duranensis Krapov. \&

\begin{tabular}{|c|c|c|}
\hline " & Fig. 6 & $20+3$ \\
\hline " & & $20+3$ \\
\hline " & Figs. $14 \mathrm{E}$ y 6 & $20+3$ \\
\hline " & Figs. $14 \mathrm{~F}$ y 6 & $20+3$ \\
\hline & Fig. 6 & \\
\hline
\end{tabular}

W.C. Gregory. Figs. 14D y 6

$20+3$
GK 10561. Paraguay, Amambay. San Luis.

K 9505. Bolivia, Santa Cruz. Cordillera, Parapetí.

$$
\begin{array}{ll}
20-5 & \text { KGBPSc 30079. Bolivia, Santa Cruz. Paja Colorada. } \\
20-5 & \text { KGBPSc 30080. Bolivia, Santa Cruz. Ipati. } \\
20-5 & \begin{array}{l}
\text { KGBPSc 30081. Bolivia, Santa Cruz. } 5 \mathrm{~km} \mathrm{~S} \mathrm{de} \\
\text { Lagunillas. }
\end{array} \\
20-6 & \text { KGBPSc 30082. Bolivia, Santa. Cruz. Ipati. } \\
20-9 & \text { KGSPSc 35005. Bolivia, Beni. Trinidad. }
\end{array}
$$

KSSc 36015. Bolivia, Santa Cruz. 2 km SW de Roboré.

$20+5$ KSSc 36021. Bolivia, Santa Cruz. Santiago.

$20+3$ ScBo 21198. Paraguay, Alto Paraguay, Cerro León, Laguna Tareyí.

$20+3$ KCP 12593. Paraguay, Ypacarai, Cerro Peró.

$20+3$ TCaVnZm 2018. Argentina, Corrientes. $11 \mathrm{~km} \mathrm{NW}$ de Santa Rosa.

$20+6$ KG 30005. Brasil, MS. Lagoa Gaiba, Jaguaribe.

$20+6$ VSGrCu 6330. Brasil, MT. $62 \mathrm{~km}$ SW of Cuiabá on road to Poconé, at rio Bento Gomes.

$20+3$ KMoF 19490. Argentina, Salta. Campo Durán (=K7988).

KScGl 28458. Argentina, Salta. Dep. Capital, San Luis, rio Arenales (=GKP 10038).

KGBPSc 30060. Argentina, Jujuy. Dep. El Carmen, rio Perico.

KGBPScS 30061. Argentina, Jujuy. Dep. Capital. 2 km $S$ de Palpalá.

KGBPScS 30064. Argentina, Jujuy. Dep. El Carmen, aeropuerto El Cadillal.

KGBPScS 30065. Argentina, Salta. Dep. S. Martín, rio Seco, Senda Hachada. 
" Fig. 6

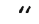

" Figs. 14G y 6

$20+3$

$20+5$

$20+5$

$20+5$

" Figs. $14 \mathrm{H} \mathrm{y} 6$

$20+5$

$20+3$

$20+3$

$20+3$

$20+3$

$20+3$

$20-3$

A. glandulifera Stalker

Figs. 13C, D y 4

A. helodes Mart. ex Krapov

\& Rigoni

" Figs. 5 y $15 \mathrm{~A}$

" Fig. 5

A. Hoehnei Krapov. \& W.C. Gregory

A. hypogaea L.ssp. fastigiata Waldron var. fastigiata. Figs. 16A y 8

A. hypogaea ssp. hypogaea var. hypogaea. Fig. 15G

A. hypogaea ssp. hypogaea var. hirsuta Köhler

Figs. 16B y 8

$20-3$

$20+5$

$20+5$

$20+5$

$20-3$

$40+3$

$40+5$
$20+3$

KGBPScS 30067. Argentina, Salta. Dep. S. Martín, Senda Hachada, rio Seco, puente Ruta 34.

KGBPScS 30069. Bolivia, Tarija. $30 \mathrm{~km} \mathrm{~N}$ de Yacuiba, Caiza.

KGBPScS 30071. Bolivia, Tarija. $2 \mathrm{~km} \mathrm{~W}$ de Saladillo.

KGBPScS 30072. Bolivia, Tarija. 14 km N de Carapari.

KGBPScS 30074. Bolivia, Tarija. 2 km N de Palmar Grande.

KGBPScS 30075. Bolivia, Tarija. 5 km S de Villa Montes.

KGBPScS 30078. Bolivia, Chuquisaca. Carandayti.

KSSc 38904. Argentina, Salta. Dep. San Martín, Dragones.

KSSc 38905. Argentina, Jujuy. Dep. Ledesma, Fraile Pintado.

Sc 15101. Paraguay, Alto Paraguay. Mayor P. Lagerenza.

ScKAl 19539. Argentina, Jujuy. Ledesma, Fraile Pintado.

ScVn 21763. Argentina, Salta. Capital, Salta. Sporting Club.

ScVn 21770. Argentina, Salta. Anta.

KGSSc 30098. Bolivia, Santa Cruz. 21 km

$\mathrm{S}$ de San Ignacio.

KGSSc 30100. Bolivia, Santa Cruz. 23 km N de San Ignacio.

KG 30036. Brasil, MT. 11 km E de Cáceres, camino a Cuiabá.

VSGrCu 6325. Brasil, MT. Santo Antonio de Leverger.

VSGrCu 6326. id.

KG 30006. Brasil, MS. Baia Vermelha, Fazenda Sta. Tereza.

K 24543. Argentina, cv.'Colorado Manfredi'.

K 33922, Brasil, Açu River, P-32 (PI-262130)

"Nambiquarae".

$40+5$ K. 34260. Perú, Bonavia 4, Criollo Blanco de Casma. 
A. hypogaea ssp. hypogaea $\quad 40+5 \quad$ K 34261. Perú, Bonavia 6, Lima.

var. hirsuta. Fig. 8

A. ipaïnsis Krapov. \&

$20-6$

W.C. Gregory. Figs. 138 y 4

$20-6$

KGBPScS 30076. Bolivia, Tarija. Ipa.

A. aff. Kempff-Mercadoi

$20+7$

Krapov. \& W.C. Gregory

A. Kuhlmannii Krapov.

\& W.C. Gregory

" Figs. 5, 15C y D

$20+7$

$20+6$

Figs. 5 y $15 B$

$20+5$

" Fig. 5

$20+5$

" Fig. 5

$20+5$

$20+5$

$20+5$

A. aff. Kuhlmannii

Krapov. \& W.C. Gregory

$20+5$

$20+5$

Fig. 5

A. magna Krapov. \& W.C.

$20-6$

Gregory. Figs. 13E y 4

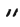

$20-6$

" Fig. 4

$20-6$

A. monticola Krapov. \& Rigoni $40+3$ Figs. $16 \mathrm{C}$ y 8

"Fig. 16D

$40+3$

"Fig. 8

$40+5$

A. stenosperma Krapov. \& W.

C. Gregory. Figs. 15E y 7
KGBPScS30090. Bolivia, Santa Cruz. Ascención Guarayos.

KG 30008. Brasi\}, MS. 150 km E de Corumbá, Fazenda Nhu Mirim.

KG 30017. Brasil, MT. $25 \mathrm{~km}$ W de Aquidauana.

KG 30035. Brasil, MT. 94 km E de Cáceres, camino a Cuiabá.

VSGr 6344. Brasil, MT. Faz. Santo André, $20 \mathrm{~km}$ SSW de Cáceres.

VSGr 6404. Brasil, MT. 56 km NW de Pto. Espiridião, BR-174 km 148.

VSGr 6410. Brasil, MT. Cáceres.

VPoJSv 10506. Brasil, MT. Mun. N. Sra. do Livramento, rio Piraim.

VPoJSv 10420. Brasil, MS. Estrada rio Negro, Aquidauana, $24 \mathrm{~km} \mathrm{NE}$ do rio Taboco.

VSGr 6380. Brasil, MT. Vila Bela.

VSGr 6413. Brasil, Mato Grosso. $45 \mathrm{~km} \mathrm{~N}$ of Cáceres on road to Barra do Bugres.

KGSSc 30092. Bolivia, Santa Cruz. El Carmen, 42 km SSW de Concepción.

KGSSc 30093. Bolivia, Santa Cruz. Embocada del Carmen, $40 \mathrm{~km}$ SSW de Concepción.

KGSSc 30097. Bolivia, Santa Cruz. San Ignacio.

K 8012. Argentina, cult. Manfredi (Jujuy. Yala).

KGBPScS 30062. Argentina, Jujuy. Yala.

KGBPScS 30063. Argentina, Jujuy. Lozano. 
A. valida Krapov. \& W.C. $\quad 20-8$ Gregory. Figs. 13F y 4

"Figs. 13G y $4 \quad 20-6$

A. villosa Benth.

Figs. $15 F$ y 7
$20+3$

$20+3$

$20+3$

$20+3$
KG 30011. Brasil, MS. 35 km SE de Corumbá, Fazenda Vale do Paraiso, Baia Negra.

KGPSc 30147. Brasil, MS. Fazenda Vale do Paraiso, Baia Negra.

Bu 22585. Argentina, Entre Ríos. Santa Ana.

HLKOj 357. Argentina, Corrientes. Paso de los Libres. K 6587. Cult. leg. J. Báez. Uruguay, Colonia.

KC 11311. Argentina, Corrientes. Monte Caseros, río Miriñay y río Uruguay.
Al principio fue difícil obtener preparados mitóticos de buena calidad para estudiar la morfología de los cromosomas. Después de varios intentos se logró desarrollar una técnica por medio de la cual se obtuvieron preparados con los cromosomas perfectamente separados, adecuados para estudiar su morfología. Esta técnica consiste en lo siguiente:

1.- Hacer germinar las semillas en potes con vermiculita a $25^{\circ} \mathrm{C}$. Una vez germinadas y cuando la raíz primaria tenga alrededor de 3 $\mathrm{cm}$, cortar el ápice radical. Las células de este ápice generalmente contienen muchas inclusiones que dificultan la observación de los cromosomas.

2.- Poner nuevamente las semillas en vermiculita. Después de 3 ó 4 días, 3 horas antes del pretratamiento, poner los potes en estufa a $35^{\circ} \mathrm{C}$.

3.- Pretratamiento. Sacar las raicillas secundarias, lavarlas bien, cortarlas y poner en 8oxiquinoleína $(0,002 \mathrm{M})$ durante 3 horas a $25^{\circ} \mathrm{C}$.

Para que los cromosomas se separen perfectamente son indispensables dos condiciones: a) El pretratamiento se debe realizar entre las 15 y 17 horas, no más tarde porque la mayoría de las células se encuentran en anafase, ni antes de las 15, porque los cromosomas están aglutinados en metafase. b) Es muy importante que exista una diferencia de 7 a $10^{\circ} \mathrm{C}$ entre la temperatura de la estufa y del pretratamiento.

4.- Fijación. Transferir las raicillas de 8oxiquinoleína a una mezcla de 5 partes de etanol absoluto y una de ácido láctico (Fernández, 1973), durante 2 a 24 horas en refrigerador.

5.- Conservación. En etanol $70^{\circ}$ en refrigerador.

\section{6.- Coloración con Feulgen.}

La descripción de los cariotipos se realizó usando la nomenclatura sugerida por Levan et al. (1964). Los cromosomas de las especies estudiadas se pudieron dividir en tres grupos: cromosomas metacéntricos $(\mathrm{m})$ (índice centromérico $=\mathrm{IC}=$ brazo corto $\times 100 /$ longitud total del cromosoma $=50-37,5$ ), submetacéntricos (sm) $(\mathrm{IC}=37,5-25)$ y subtelocéntricos (st) (IC=25-12,5).

La ordenación de los cromosomas en los cariotipos se hizo colocando en primer término los cromosomas metacéntricos seguidos por los submetacéntricos y subtelocéntricos. Dentro de cada grupo, los cromosomas se dispusieron en forma decreciente de acuerdo con su tamaño. Las mediciones de los cromosomas se hicieron sobre la base de dibujos de metafases donde los cromosomas estaban en un solo plano. La cantidad de metafases necesarias para dejar establecido el cariotipo de una especie, depende de la variabilidad de los cromosomas que puedan presentarse en las distintas metafases. Por ejemplo, en $A$. ipaënsis se encontró en cada una de las siete metafases analizadas: 9 pares metacéntricos y 1 par submetacéntrico. En este caso se considera que las siete metafases son suficientes para dejar establecido el cariotipo de esta especie. Siguiendo este criterio se han confeccionado 45 cariotipos que corresponden a 22 especies y 4 subespecies las que represen$\tan 5$ de las 9 secciones del género Arachis; ellas son: Trierectoides, Erectoides, Caulorrhizae, Procumbentes y Arachis.

En muchos casos los preparados no fueron suficientes para elaborar los cariotipos, pero fueron adecuados para analizar la morfología de los cromosomas "A" y SAT de 41 especies. 


\section{Resultados}

Los cromosomas de Arachis pertenecen a la categoría de pequeños, de acuerdo con la clasificación de Lima-De-Faria (1980). El más grande mide $2,2 \mu \mathrm{m}$ en A. Batizocoi y $4,05 \mu \mathrm{m}$ en $A$. stenosperma, y el más pequeño mide 1,11 $\mu \mathrm{m}$ en $A$. duranensis (30064) y $1,86 \mu \mathrm{m}$ en $A$. stenosperma.

En general, todos los cromosomas son muy semejantes entre sí, a excepción de dos pares que se pueden diferenciar perfectamente: un par con constricción secundaria y un par pequeño, cromosomas SAT y " $A$ ", respectivamente (Husted, 1936). Estos pares de cromosomas se han estudiado en detalle en 41 especies y 4 subespecies pertenecientes a 8 secciones de las 9 que posee el género Arachis (Tabla 2).

En todas las especies estudiadas hay una condensación diferencial de los cromosomas en profase. La condensación de los cromosomas comienza por la región proximal y continúa hacia los extremos. En esta fase se nota una coloración intensa en las regiones proximales y débil en las distales. A. hypogaea ssp. hypogaea (Nambiquarae) (Fig. 15G) presenta un par de cromosomas con una coloración menos intensa en las regiones media y distal de los dos brazos, siendo la coloración más intensa en las regiones proximal y subdistal. Pocas especies presentan un par de cromosomas, como máximo, teñido casi totalmente. En metafase, todos los cromosomas muestran una coloración uniforme, no observándose zonas menos teñidas.

Otra de las características de los cromosomas de Arachis consiste en que hay especies que tienen uno, dos y hasta tres pares de cromosomas con el centrómero inusualmente estirado en profase y prometafase. En $A$. Cardenasii (21198 y 36021), A. helodes, A. valida (30011), A. aff. duranensis (15101) y A. correntina (2018) se encuentra un par con este tipo de centrómero. En A. Cardenasii (36015) se presentan tres pares lo que se deduce de la presencia de hasta 28 elementos. En la Fig. 14A se observan 27 elementos. Estos cromosomas tan peculiares pueden causar confusión al tratar de establecer el número cromosómico. Esta condición no se puede atribuir a la presión que se realiza al aplastar el material, ya que en todas las especies se aplicó más o menos la misma presión, y solamente aparece en las especies mencionadas y en todas las células. El centrómero extendido fue observado anteriormente en especies de otros géneros, como Antirrhinum majus, Allium sphaerocephalum (Lima-De-Faria, 1956) y Libocedrus chilensis (Hunziker, 1961).

Se han observado células polisomáticas en A. correntina, A. duranensis (30064), Arachis paraguariensis ssp. paraguariensis (30110), A. major (10582, 14444 y 30128) y A. monticola (30062). En esta última especie se encontró mayor cantidad de células polisomáticas $(2 n=80$, Fig. 16D) que de células normales $(2 n=40)$, en la accesión 30062; sin embargo, no se han observado en las accesiones 8012 y 30063 . En $A$. duranensis (30064) que posee en células normales $2 \mathrm{n}=20$, se observaron células polisomáticas con $2 n=40$ y $2 n=80$ cromosomas.

\section{Cromosomas SAT}

Todas las especies estudiadas poseen un par de cromosomas con constricción secundaria, excepto una accesión (30147) de A. valida donde se encontraron dos pares de cromosomas con constricción secundaria. Sobre la base del tamaño relativo del satélite y la posición del centrómero, los pares SAT se han podido clasificar en 10 tipos (Tabla 2 y Fig. 10).

Para facilitar la descripción de los cromosomas SAT, se los dividió en brazo 1 y brazo 2, separados por el centrómero (Fig. 1). Al brazo 2 se lo dividió a su vez en segmento proximal y segmento distal (satélite).

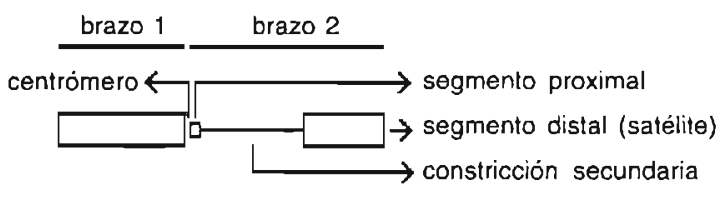

Fig. 1. Morfología del cromosoma SAT.

Los 10 tipos de cromosomas con constricción secundaria tienen las siguientes características:

Tipo 1. El satélite es más pequeño que el brazo 1, pero más grande que el segmento proximal. 
Tabla 2. Especies de Arachis con cromosomas SAT (tipos 1 a 10) y presencia de cromosonas "A".

\begin{tabular}{|c|c|c|c|c|c|c|c|c|c|c|c|c|}
\hline$\square[-\square$ & $\square-\square_{2}$ & $\square \square \square$ & $\square \square$ & {$\left[\begin{array}{r}\square \\
5\end{array}\right.$} & $\square-\square$ & $\frac{\square}{\square-\square}-\frac{\square}{3}$ & 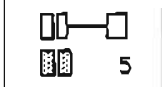 & $\begin{array}{cc}0 & -7 \\
6 & 6\end{array}$ & 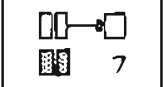 & {$[\square-10$} & $\begin{array}{r}\square \square \\
9\end{array}$ & $\square \square \longrightarrow$ \\
\hline $\begin{array}{l}\text { EXTRAMERUOSAE } \\
\text { uillosulicarpa }\end{array}$ & $\begin{array}{l}\text { TRIERECTOIDES } \\
\text { guaranitica } \\
\text { ERECTOIDES } \\
\text { najor } 8 \\
\text { Benthamii } 2 \\
\text { gracilis } \\
\text { Archeri } \\
\text { Hernannii } \\
\text { EXTRALERULSAE } \\
\text { aff. prostrata } \\
\text { uillosulicarpa } \\
\text { HETERANTHAE } \\
\text { Dardani } \\
\text { pusilla } \\
\text { CAULORRHIZAE } \\
\text { Pintoi }\end{array}$ & $\begin{array}{l}\text { ERECTOIDES } \\
\text { paraguariensis } \\
\text { ssp. capib, } 5 \\
\text { ARACHIS } \\
\text { Hoehnei } \\
\text { glandulifera } 2 \\
\text { EXTrakERUOSAE } \\
\text { lutescens } \\
\text { Burchellii } \\
\text { Pintoi }\end{array}$ & $\begin{array}{c}\text { ERECTOIDES } \\
\text { paraguariensis } \\
\text { s5p. parag. II } \\
\text { stenophylla } 3\end{array}$ & $\begin{array}{l}\text { ARACHIS } \\
\text { 8atizQCOI } 4\end{array}$ & $\begin{array}{l}\text { ARACHIS } \\
\text { Batizocol } \\
\text { ipaensis } 2 \\
\text { magna } 3\end{array}$ & $\begin{array}{l}\text { ARACHIS } \\
\text { Cardenasii } 2 \\
\text { duranensis 13 } \\
\text { Kuhldannii } \\
\text { correntina } 2 \\
\text { stenosperma } \\
\text { villosa } 4\end{array}$ & $\begin{array}{l}\text { ARACHIS } \\
\text { Cardenasii } \\
\text { duranensis } 6 \\
\text { Kuhimannii } 5 \\
\text { aff.KuhImannis } 2 \\
\text { helodes } 5\end{array}$ & $\begin{array}{l}\text { ARACHIS } \\
\text { Diogoi } 2 \\
\text { Xuhimannii }\end{array}$ & $\begin{array}{l}\text { ARACHIS } \\
\text { Kempif-Kercadoi } \\
\text { Xuhlmannii }\end{array}$ & $\begin{array}{l}\text { ARACHIS } \\
\text { valida }\end{array}$ & $\begin{array}{l}\text { ARACHIS } \\
\text { benensis } \\
\text { ProcumBEMTES } \\
\text { Rigonli } \\
\text { lignosa } \\
\text { aatiensis } 4 \\
\text { aff. matiensis } \\
\text { appressipila } 3 \\
\text { Kretschmeri } 2\end{array}$ & $\begin{array}{l}\text { METERAMTHAE } \\
\text { syluestris } 5 \\
2 n=2 x=20\end{array}$ \\
\hline & & $\begin{array}{l}\text { RHIZOMATOSAE } \\
\text { glabrata var. } \\
\text { HagenbecXil }\end{array}$ & & & & \begin{tabular}{|l|} 
onticola 2 \\
(Yala) \\
hypogaea \\
var. fastigiata
\end{tabular} & $\begin{array}{l}\text { monticola } \\
\text { (Lozano) } \\
\text { hypogaea } \\
\text { uar. hypogaea } \\
\text { "nambyquarae" } \\
\text { hypogaea } \\
\text { var. hirsuła } 2\end{array}$ & & & & & $2 n=4 x=40$ \\
\hline
\end{tabular}


Tipo 2. El satélite es más pequeño que el brazo 1 y el segmento proximal es diminuto $(0,2 \mu \mathrm{m})$ (Fig. 11B profase, C metafase).

Tipo 3. El satélite es más o menos igual al brazo 1 más el segmento proximal, el cual es diminuto $(0,2 \mu \mathrm{m})$. En profase el brazo 1 tiene igual coloración que el satélite (Fig. 11E).

Tipo 4. Igual al tipo 3 en cuanto a la forma y tamaño, pero el brazo 1 en profase y en prometafase presenta coloración menos intensa que el satélite (Fig. 11D).

Tipo 5. El satélite tiene más o menos el mismo tamaño que el brazo 1 más el segmento proximal. El segmento proximal es algo menor que el brazo 1 (Fig. 13A).

Tipo 6. El satélite es algo mayor que el brazo 1 y éste es más grande que el segmento proximal (Fig. 13E).

Tipo 7. Satélite doble. El satélite terminal más el intercalar tienen casi el mismo tamaño que el brazo 1 más el segmento proximal.

Este cromosoma tiene dos constricciones secundarias, una entre el segmento proximal y el satélite intercalar y otra entre éste y el satélite terminal (Fig. 15C y D).

Tipo 8. El satélite es la mitad en tamaño que el brazo 1. El brazo 1 es algo mayor que el segmento proximal (Fig. 13F).

Tipo 9. El satélite es punctiforme y su tamaño es igual o un poco más pequeño que el segmento proximal. El brazo 1 es mayor que el segmento proximal (Fig. 12C).

Tipo 10. El satélite es punctiforme, pero más pequeño que el anterior. El brazo 1 es más o menos igual al segmento proximal (Fig. 12A).

Los cromosomas de los tipos 1 a 7 poseen macrosatélite (satélite lineal para Battaglia, 1955) y los tipos 9 y 10 poseen microsatélite (Battaglia, 1955). El tipo 8 es intermedio entre el macro y microsatélite (macrosatélite para Battaglia, 1955).

El comportamiento de los cromosomas SAT es extraordinario durante la profase y hasta la metafase temprana, en este período el satélite se encuentra muy alejado del cromosoma al cual corresponde. El filamento no es fácil de observar, salvo en $A$. glandulifera (Fig. 13D) y A. hypogaea ssp. hypogaea var. hirsuta, Bonavia 4 (Fig. 16B). Este alargamiento de la constricción secundaria, es una característica propia del género Arachis, y no influye sobre él ni el aplastado, ni el pretratamiento, ni el fijador utilizado. Esta característica ya había sido observada por Husted (1936) en $A$. hypogaea en preparados sin pretratamiento. En preparados sin pretratamiento, también se pudo observar la constricción secundaria muy alargada en A. Batizocoi (Fig. 13A).

En la anafase de $A$. glandulifera se pudo diferenciar perfectamente la constricción primaria de la secundaria y, por tanto, la ubicación del satélite (Fig. 13D).

El par de cromosomas con macrosatélite, del tipo 4, que poseen las especies de la sección Erectoides tiene peculiar comportamiento en dos taxones (A. stenophylla y $A$. paraguariensis ssp. paraguariensis). En profase, el brazo 1 y el segmento proximal están difusamente teñidos, mientras que el satélite ya ha adquirido una coloración intensa, como se observa en la ssp. paraguariensis (30124) (Fig. 11D), manteniéndose estas condiciones hasta la metafase. En metafase ya no se pueden distinguir los cromosomas con macrosatélite del tipo 4 de los del tipo 3. La diferencia entre estos dos tipos se determina por el comportamiento en profase. Los cromosomas con satélite del tipo 4 que se observan en $A$. paraguariensis ssp. paraguariensis (30110) son metacéntricos, mientras que en la accesión 30115 y en A. stenophyla (572 y 30126) son submetacéntricos.

En algunas especies el segmento proximal es diminuto, lo que a veces dificulta la individualización en metafase del par de cromosomas con satélite como en $A$. villosa, $A$. correntina, A. lutescens y A. guaranitica (Fig. 11A). Esta sería la causa por la cual Stalker \& Dalmacio (1981) no observaron cromosomas con satélite en $A$. villosa, $A$. correntina y $A$. duranensis (sub A. Spegazzinii). Sin embargo, según la experiencia de un autor del presente trabajo (A.F.), en la foto de los cariotipos de $A$. villosa y A. correntina publicados por los citados investigadores el par 2 correspondería a los cromosomas con satélite.

\section{Cromosoma "A"}

El par " $A$ " observado por primera vez por Husted (1933 y 1936) en A. hypogaea y posteriormente por Krapovickas \& Rigoni (1951), Smartt (1964), Stalker \& Dalmacio (1981) y 
Singh \& Moss (1982) en especies silvestres pertenecientes a la sección Arachis, también fue observado en el presente trabajo exclusivamente en especies de esta misma sección. Ella contiene especies sin el par "A", como $A$. Batizocoi (Krapovickas et al., 1974; Smartt et al., 1978; Stalker \& Dalmacio, 1981; Singh \& Moss, 1982 y Stalker et al., 1991a), A. ipaënsis, A. magna, $A$. valida, A. glandulifera, A. benensis y $A$. Hoehnei. El tamaño del par "A" en metafase es un poco menor que la mitad del par más grande, salvo en $A$. helodes y $A$. Cardenasii, donde es algo mayor ( $54 \%$ ) que la mitad de dicho par. Esto último a veces dificulta la individualización del par " $\mathrm{A}$ " en metafase. En las especies que no tienen el par diminuto, el tamaño del cromosoma menor es superior al $65 \%$ del más grande.

En profase, en cambio, el par " $\mathrm{A}$ " es fácilmente individualizable, debido a que su coloración es menos intensa que la del resto, como se puede observar en $A$. correntina (Fig. 14C), A. Kuhlmannii (Fig. 15C)y A. hypogaea ssp. hypogaea var. hypogaea (Fig. 15G). Stalker \& Dalmacio (1981) no observaron este par en $A$. Cardenasii.

El par " $A$ " se comporta en forma diferente de los demás cromosomas durante el ciclo celular. En profase está levemente teñido, excepto en la región proximal, donde la coloración es más intensa, y prácticamente se mantiene esta condición hasta la prometafase. Recién en metafase media todos los cromosomas están total y uniformemente teñidos. En este estado se han seleccionado las metafases para determinar los cariotipos.

El par " $A$ " es un buen marcador genético, ya que en cruzamientos interespecíficos entre especies que lo poseen se producen híbridos con viabilidad de polen superior a los híbridos entre especies con el par " $\mathrm{A}$ " o sin él ( Raman \& Kesavan, 1965; Smartt et al., 1978; Resslar \& Gregory, 1979; Raman, 1981; Singh, 1986; Stalker, 1991; Stalker et al., 1991b).

Utilizando el par SAT y la presencia o ausencia del par " $A$ " se ha confeccionado la Tabla 2. La misma está separada por dos líneas; en la parte superior figuran las especies diploides y en la inferior las tetraploides. A la derecha de cada especie se menciona la cantidad de accesiones estudiadas, cuando son más de una.
En la Fig. 9 se muestra la distribución geográfica de estos dos pares de cromosomas. Los números representan los diferentes tipos de cromosomas SAT y los encerrados con un círculo poseen además el par " $A$ ".

\section{Cariotipo}

En la Tabla 3 se presentan las características cromosómicas de las especies cuyos cariotipos se determinaron y que se ilustran en las figuras $2,3,4,5,6,7$ y 8 .

\section{Sección Trierectoides}

A. guaranitica (Fig. 11A y 2): los 10 pares de cromosomas son metacéntricos, el macrosatélite es del tipo 2 y el promedio del índice centromérico es el valor más alto (46.63) entre todas las especies. El tamaño de los cromosomas varía entre 1.82 y $2.70 \mu \mathrm{m}$.

\section{Sección Erectoides}

A. major (Fig. 2): los cariotipos de dos accesiones, 14444 y 30128 , presentan los 10 pares metacéntricos, teniendo el par 3 macrosatélite del tipo 2, mientras que el cariotipo de la accesión 555a (Fig. 11B y C) presenta 8 pares metacéntricos y 2 pares submetacéntricos y macrosatélite también del tipo 2. El tamaño de los cromosomas varía entre 2.16 (555a) y 3.62 $\mu \mathrm{m}$ (14444).

A. paraguariensis (Fig.2): se estudiaron dos accesiones de la subespecie paraguariensis y una de la subespecie capibarensis. Las tres accesiones presentaron cariotipos diferentes. La accesión 573, ssp. capibarensis (Fig. 11E y F), posee 9 pares de cromosomas metacéntricos y 1 par submetacéntrico, el par de cromosomas con macrosatélite es del tipo 3 y el satélite está en el brazo corto. La subespecie paraguariensis (30110) posee 8 pares de cromosomas metacéntricos (1-8) y 2 pares submetacéntricos (9 y 10), el con macrosatélite es del tipo 4 y metacéntrico. La accesión 30115 posee 7 pares de cromosomas metacéntricos y 3 pares de submetacéntricos, el par con macrosatélite es del tipo 4 y submetacéntrico. El tamaño de los cromosomas varía entre $1.70 \mu \mathrm{m}$ (573) y 2.84 $\mu \mathrm{m}$ (30110). La Fig. 11D es una profase de la 
BONPLANDIA 8 (1-4), 1994

Sección Trierectoides

$\begin{array}{llllllllll}1 & 2 & 3 & 4 & 5 & 6 & 7 & 8 & 9 & 10\end{array}$

A. guaranitica (10568)

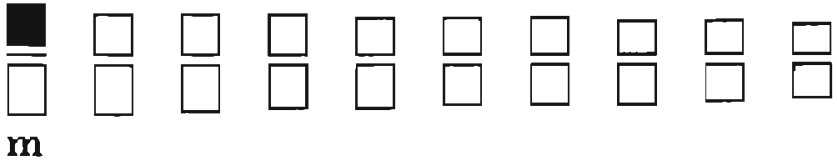

Sección Erectoides

A. major (555a)

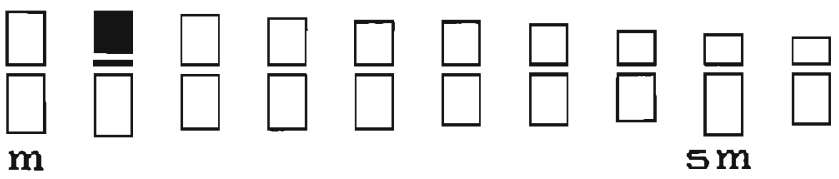

A. major (14444)

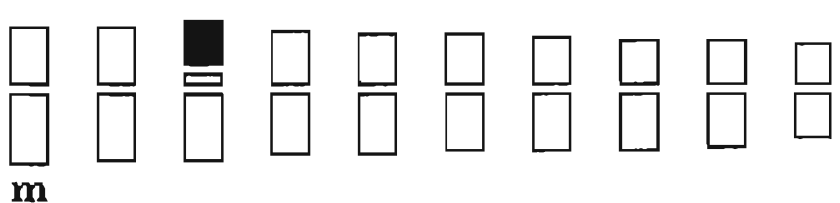

A. major (30128)

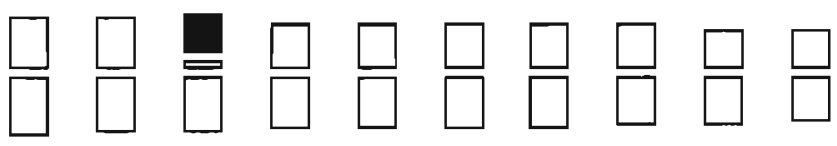

m

A. paraguariensis (573)
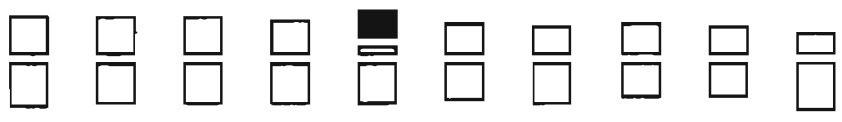

m

$5 \mathrm{~m}$

A. paraguariensis (30110) ssp. paraguariensis
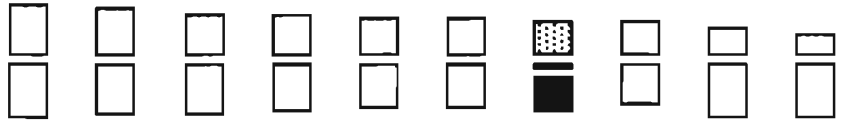

$5 \mathrm{~m}$

A. Paraguariensis (30115) Ssp. paraguariensis
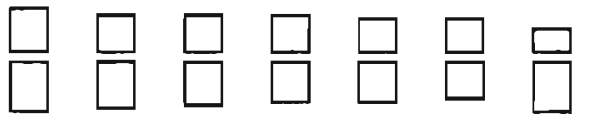

$5 \mathrm{~m}$

m

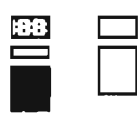

A. stenophylla (572)

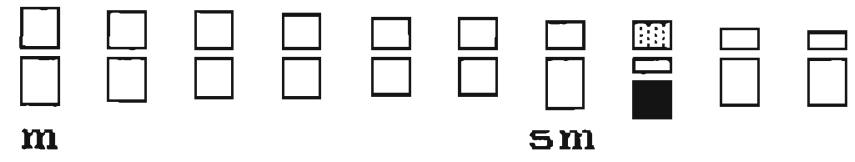

A. stenophylla (30126)

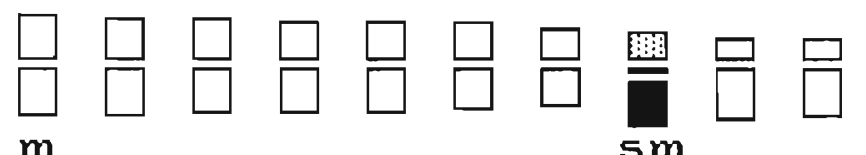

Fig. 2. Cariotipos de especies de Aricints. Escala igual a $5 \mu \mathrm{m}$. 
ssp. paraguariensis (30124), donde se observa la característica del cromosoma SAT del tipo 4.

A. stenophylla (Fig. $11 G$ y 2): se estudiaron dos accesiones. En la 572 se observaron 6 pares metacéntricos y 4 pares submetacéntricos, el par 8 con macrosatélite del tipo 4; en la 30126 se observaron 7 pares de cromosomas metacéntricos y 3 pares de submetacéntricos. Las dos accesiones poseen el par con macrosatélite del tipo 4 . El tamaño de los cromosomas varía entre 1.80 y $2.56 \mu \mathrm{m}$ (30126).

\section{Sección Extranervosae}

Se analizó una sola célula en $A$. aff.prostrata (5913) (Fig. 13H), que posee 6 pares de cromosomas metacéntricos y 4 pares de submetacéntricos. El par de cromosomas con satélite es del tipo 2. El índice centromérico tiene un valor de 39.12. El tamaño de los cromosomas varía entre 1.58 y $2.39 \mu \mathrm{m}$, con una media de $1.84 \mu \mathrm{m}$.

\section{Sección Caulorrhizae}

Esta sección posee dos especies, de las que se estudió una, $A$. Pintoi (Fig. 12B y 3), que presenta 9 pares metacéntricos y un par submetacéntrico, con macrosatélite del tipo 2 ubicado en el par 6. El tamaño de los cromosomas varía entre 1.77 y $2.83 \mu \mathrm{m}$.

\section{Sección Procumbentes}

Las especies de esta sección se caracterizan por poseer cromosomas con satélite punctiforme del tipo 9 (Tabla 2).

A. lignosa (Fig. $12 \mathrm{C} \mathrm{y} \mathrm{3)} \mathrm{tiene} 8$ pares metacéntricos, 2 pares submetacéntricos y el par 9 con microsatélite. El tamaño de los cromosomas varía entre 1.75 y $2.77 \mu \mathrm{m}$.

En A. Rigonii (Fig. 12D) no se consiguió suficiente cantidad de metafases para establecer el cariotipo, pero dos metafases presentaron 8 pares metacéntricos y dos pares submetacéntricos.

A. Kretschmeri (30007): posee 9 pares metacéntricos (1-9) y uno submetacéntrico (par 10) con microsatélite (Fig. 12E y 3). El tamaño de los cromosomas varía entre 1.72 y $2.85 \mu \mathrm{m}$.

En $A$. appressipila (Fig. 12F) no se hizo el idiograma por disponerse de una sola célula, donde se pudieron observar 7 pares metacéntricos y 3 pares submetacéntricos. Posee un par con microsatélite tipo 9.

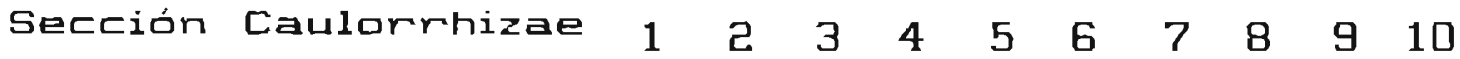 \\ A. Pintoi (6791)
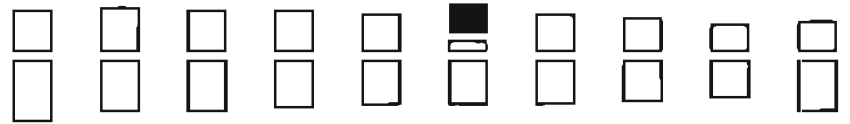 \\ m \\ Sección Procumbentes \\ A. Kretschmeri (30607) \\ A. lignosa (14248)
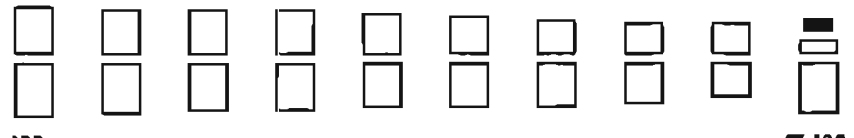 \\ $5 \mathrm{~m}$

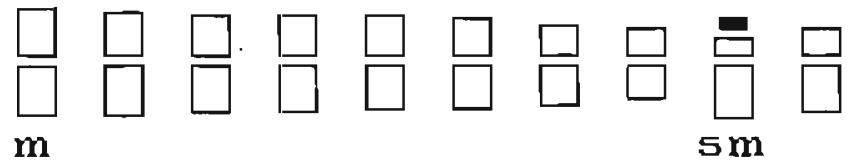

Fig. 3. Cariotipos de especies de Arachis. Escala igual a $5 \mu \mathrm{m}$. 
Sección Arachis

Las especies que se mencionan a continuación no poseen el par " $\mathrm{A}$ ".

A. Batizocoi (9505) (Fig. 13A y 4): posee 6 pares metacéntricos, 3 pares submetacéntricos y un par subtelocéntrico/submetacéntrico que corresponde al par de cromosomas con macrosatélite y es del tipo 5 . El tamaño de los cromosomas varía entre 1.71 y $2.20 \mu \mathrm{m}$.

A. ipaënsis (19455) (Fig. 13B y 4): posee 9 pares metacéntricos y uno submetacéntrico. El par 5,

Sección Arachis

A. Batizocoi (9505)

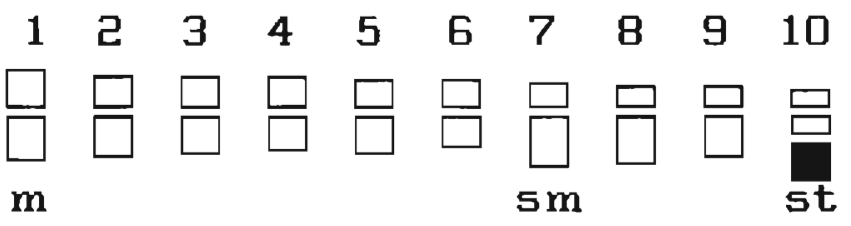

A. ipaënsis (19455)

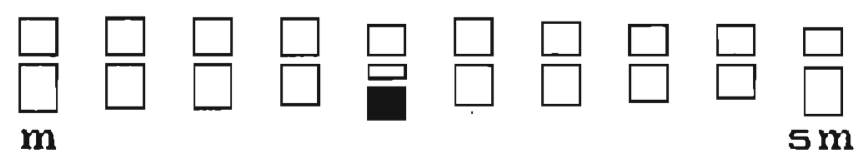

A. glandulifera (30098)

A. magina (30092)
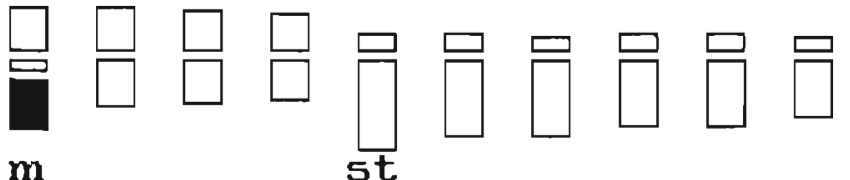

A. magna (30097)
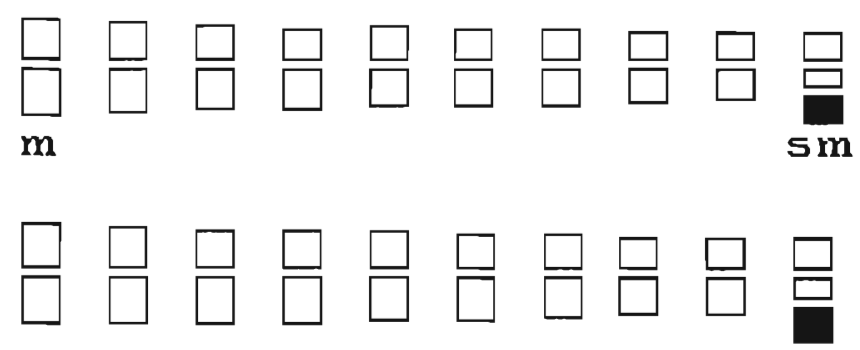

m

$5 \mathrm{~m}$

A. valida (30011)

A. valida (30147)
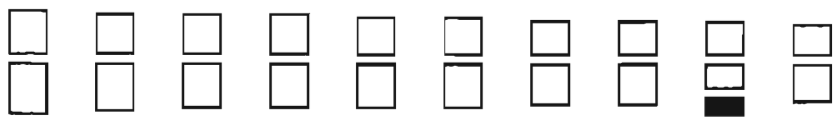

m
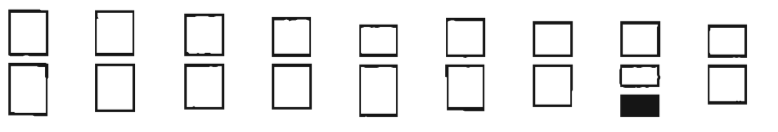

m

Fi. 4. Cariotipos de especies de Arachis secc. Arachis, sin cromosoma "A". Escala igual a 5 mm. 
con macrosatélite del tipo 6, es metacéntrico. El tamaño de los cromosomas varía entre 1.84 y $2.44 \mu \mathrm{m}$.

A. glandulifera (30098) (Fig. 13C y 4): posee 4 pares metacéntricos y 6 pares subtelocéntricos. El par de cromosomas con macrosatélite (tipo 3 ) es el más grande (par 1) de los metacéntricos, pero es más pequeño que el par 5 subtelocéntrico. La accesión 30100 aparentemente también tiene el mismo cariotipo. El tamaño de los cromosomas varía entre 2.07 y $2.98 \mu \mathrm{m}$. En la Fig. 13D se observan perfectamente los filamentos y los satélites rezagados con filamentos entrelazados en anafase.

A. magna (Fig. 4): en dos accesiones, 30092 (Fig. 13E) y 30097, se observaron 9 pares metacéntricos y uno submetacéntrico con macrosatélite del tipo 6. El tamaño de los cromosomas varía entre 1.58 (30092) y $2.56 \mu \mathrm{m}$ (30097). En la accesión 30093 se han analizado dos célúlas que presentan el mismo cariotipo que las otras dos accesiones.

A. valida (Fig. 4: presenta cariotipos diferentes en las dos accesiones analizadas. En la 30011 (Fig. 13F), los 10 pares de cromosomas son metacéntricos; el par de cromosomas con satélite (par 9) es del tipo 8. En cambio, en la 30147 (Fig. 13G), que es una colección de la misma localidad, se observan 9 pares metacéntricos y uno submetacéntrico. Lo destacable de esta última es la presencia de dos pares de cromosomas con satélite, uno (par 7) es del tipo 8 y el otro (par 10) es del tipo 6. El tamaño de los cromosomas varía entre 1.92 y $2.72 \mu \mathrm{m}$.

Las especies que se mencionan a continuación poseen el par de cromosomas " $\mathrm{A}$ ".

A. Cardenasii (36021): $2 n=2 x=20$, posee 8 pares metacéntricos y dos pares submetacéntricos. El par " $A$ " es el 8 y el macrosatélite es del tipo 5 y corresponde al par 10 (Fig. 5), mientras que en la accesión 36015 (Fig. 14A y B) el macrosatélite es del tipo 3. El tamaño de los cromosomas varía entre 1.41 y $2.74 \mu \mathrm{m}$.

A. correntina (2018): $2 \mathrm{n}=2 \mathrm{x}=20$, presenta 8 pares metacéntricos y dos pares submetacéntricos. Uno de los primeros, el par 6, corresponde al del macrosatélite (tipo 3) y el par 8 es el "A" (Figs. 14C y 5).El tamaño de los cromosomas varía entre 1.56 y $3.48 \mu \mathrm{m}$.

A. helodes (Fig. 5): $2 n=2 x=20$, el cariotipo fue analizado en dos accesiones: 6325 (Fig. 15A) y
6326. Las dos poseen 8 pares metacéntricos y dos pares submetacéntricos, en ambos el par con macrosatélite (tipo 5) es submetacéntrico y el par " $\mathrm{A}$ " es el 8. El tamaño de los cromosomas varía entre 1.28 (6326) y $2.97 \mu \mathrm{m}(6325)$.

A. Kuhlmannii (Fig. 5): $2 n=2 x=20$, se estudió el cariotipo de 4 accesiones, tres de las cuales, 6344 (Fig. 15B), 6404 y 6410, poseen 8 pares metacéntricos y dos pares submetacéntricos, con macrosatélite del tipo 5 y el par " $A$ " es el 8. La cuarta, 30017, presenta 9 pares metacéntricos y un par submetacéntrico (par 10), que corresponde al cromosoma con satélite del tipo 7 y el par 9 es el " $A$ ". Los cromosomas SAT de tipo 7 tienen la particularidad de poseer dos constricciones secundarias y dos satélites, una intercalar y la otra terminal (Fig. 15C y D). El tamaño de los cromosomas varía entre 1.33 (30017) y $3.24 \mu \mathrm{m}$ (6404).

La accesión $6413(2 n=2 x=20)$, afín a $A$. Kuhlmannii (Fig. 5), posee 9 pares metacéntricos, uno de los cuales es el par "A" (par 9) y un par submetacéntrico con macrosatélite, del tipo 5. El tamaño de los cromosomas varía entre 1.82 y $3.41 \mu \mathrm{m}$.

A. duranensis, $2 \mathrm{n}=2 \mathrm{x}=20$ (Fig. 6): los cariotipos de cuatro accesiones, 19490 (Fig. 14D), 28458, 30061 (Fig. 14E) y 30064 (Fig. 14F), no muestran diferencias, salvo en la posición de los cromosomas SAT. Estas cuatro accesiones poseen 9 pares metacéntricos y un par submetacéntrico (par 10). El par de cromosomas con macrosatélite (tipo 3) es metacéntrico, estando ubicado en el $\mathrm{N}^{\circ} 3$ en las procedencias 30061 y 30064 y en el No 5 en la 19490 y 28458. El par " $A^{\prime \prime}$ es el 9. Los cariotipos de otras cuatro accesiones: 30065, 30067, 30072 (Fig. 14G) y 30078 (Fig. 14H), no muestran diferencias entre sí, poseen 9 pares metacéntricos y un par submetacéntrico (par 10), que corresponde al cromosoma con macrosatélite del tipo 5. Estas cuatro últimas accesiones difieren de las cuatro primeras en el tipo de cromosomas con macrosatélite y en un par submetacéntrico (Fig. 6). En la accesión 19539, los diez pares de cromosomas son metacéntricos. El cromosoma SAT es del tipo 3 y está ubicado en el par 7. El par " $\mathrm{A}$ " es el 9. El tamaño de los cromosomas varía entre 1.00 (28458) y $2.95 \mu \mathrm{m}$ (30078).

A. Diogoi (6330), $2 n=2 x=20$ : presenta 9 pares metacéntricos y un par submetacéntrico (Fig. 
Sección Arachis

$\begin{array}{llllllllll}1 & 2 & 3 & 4 & 5 & 6 & 7 & 8 & 9 & 10\end{array}$

A. Cardenasii (36021)

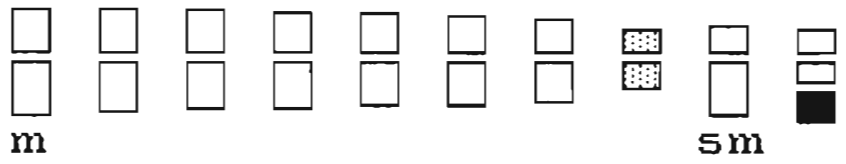

A. correntina (2018)

A. helodes (6325)

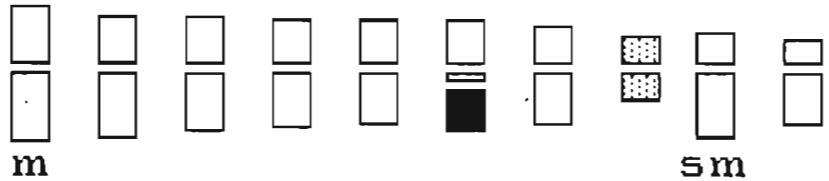

$\square \square \square \square \square \square \square \square \underset{\mathbf{m}}{\square} \square \square$

A. helodes (6326)

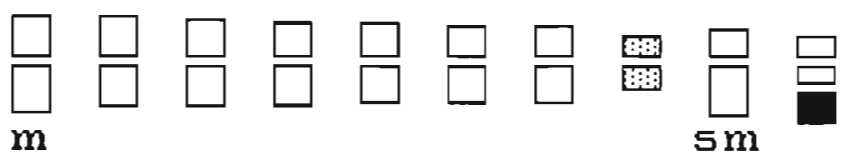

A. Kuhlmannii (6344)

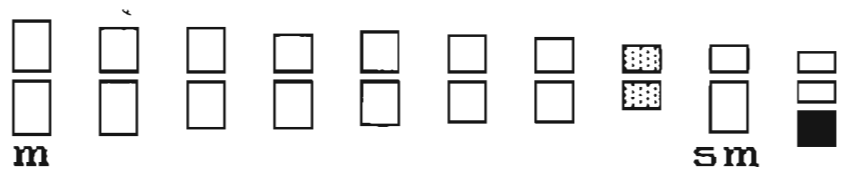

A. Kuhlmannii (6404)

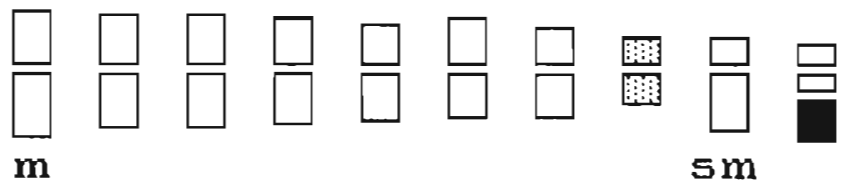

A. Kuhlmannii (6410)

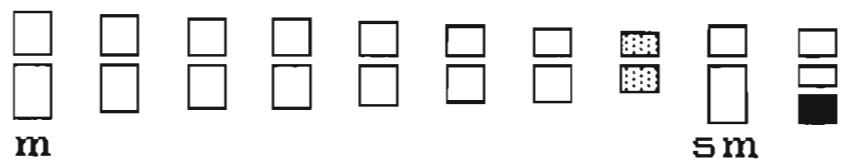

A. Kuhlmannii (3ø017)
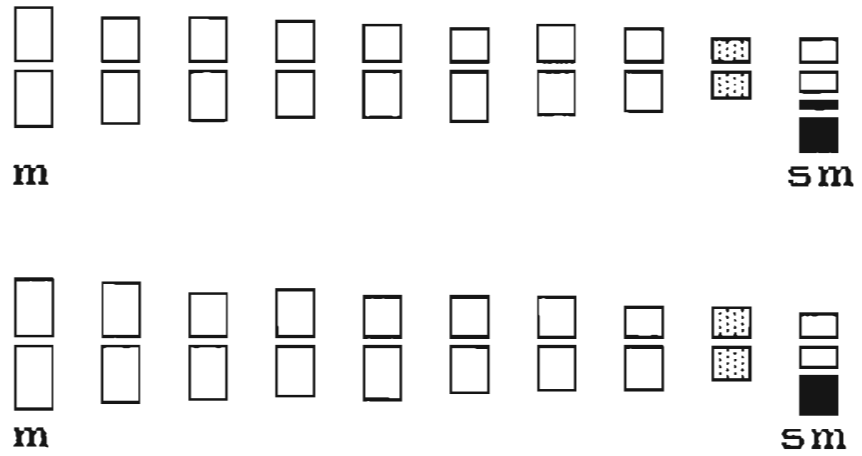

Fig. 5. Cariotipos de especies de Arachis secc. Arachis, con cromosoma "A". Escala igual a $5 \mu \mathrm{m}$. 
Sección Arachis A. duranensis (19539)

(19490)

(28458)

(30061)

(30064)

$(30065)$

$(30067)$

(30072)

(30078) $\begin{array}{llllllllll}1 & 2 & 3 & 4 & 5 & 6 & 7 & 8 & 9 & 10\end{array}$

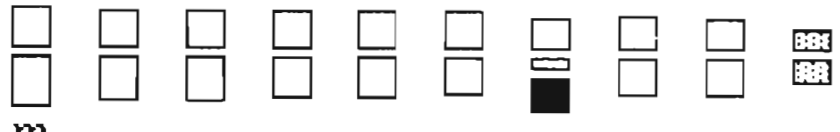

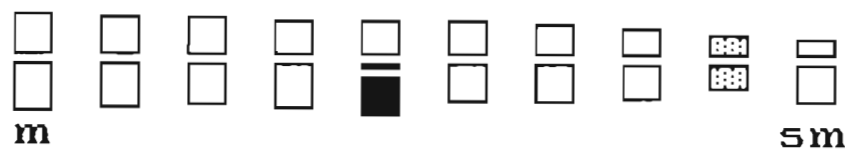

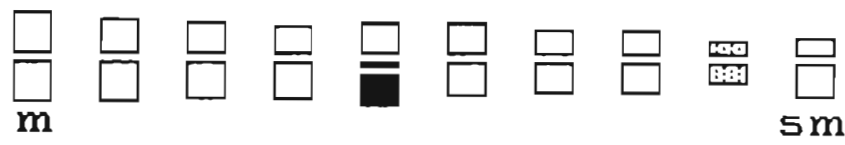

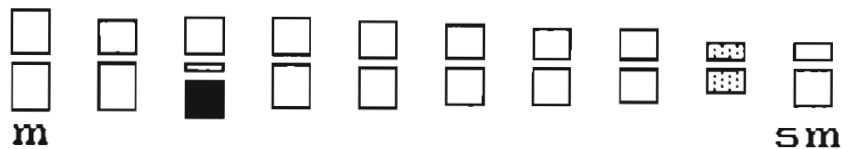

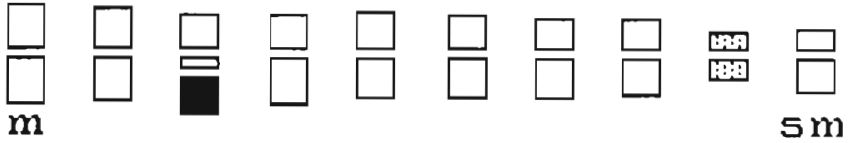

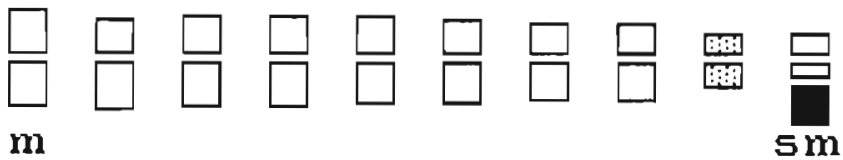

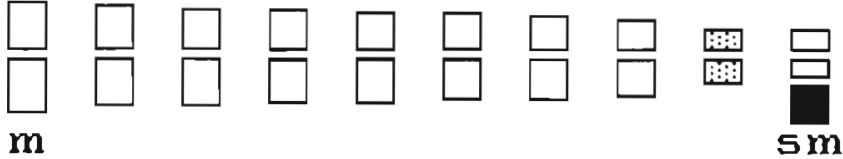

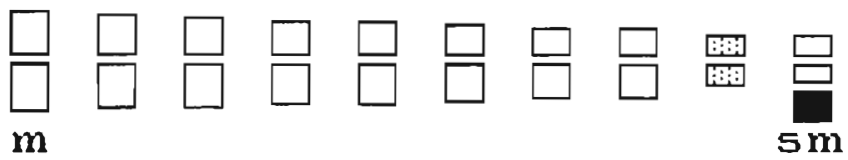

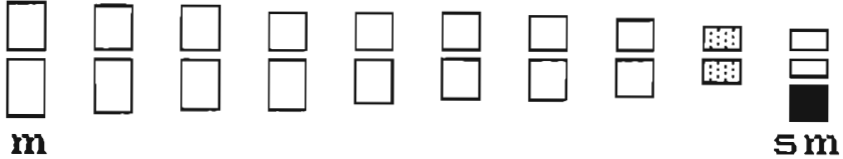

Fig. 6. Cariotipos de A. duranensis, con cromosoma "A". Escala igual a $5 \mu \mathrm{m}$. 
BONPLANDIA 8 (1-4), 1994

Sección Arachis

$\begin{array}{llllllllll}1 & 2 & 3 & 4 & 5 & 6 & 7 & 8 & 9 & 10\end{array}$

A. Diagai (6330)

$\begin{array}{lllllllllll}\square & \square & \square & \square & \square & \square & \square & \square & \square & \square\end{array}$

A. stenosperma (410)

A. villosa (357)

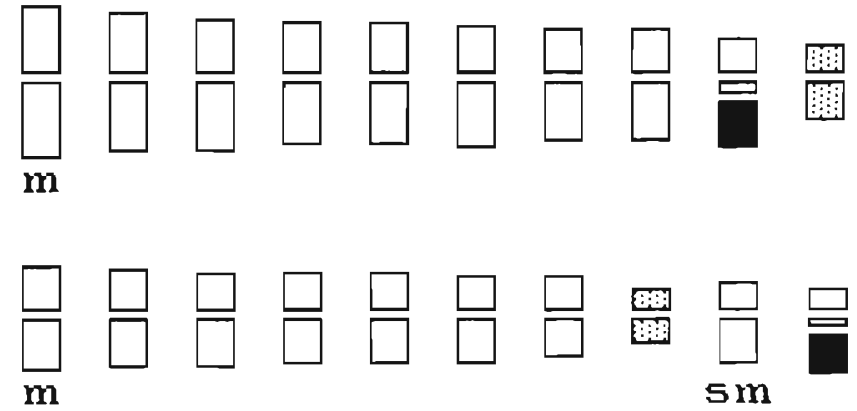

Fig. 7. Cariotipos de especies de Arachis secc. Arachis, con cromosomas "A". Escala igual a 5 um.
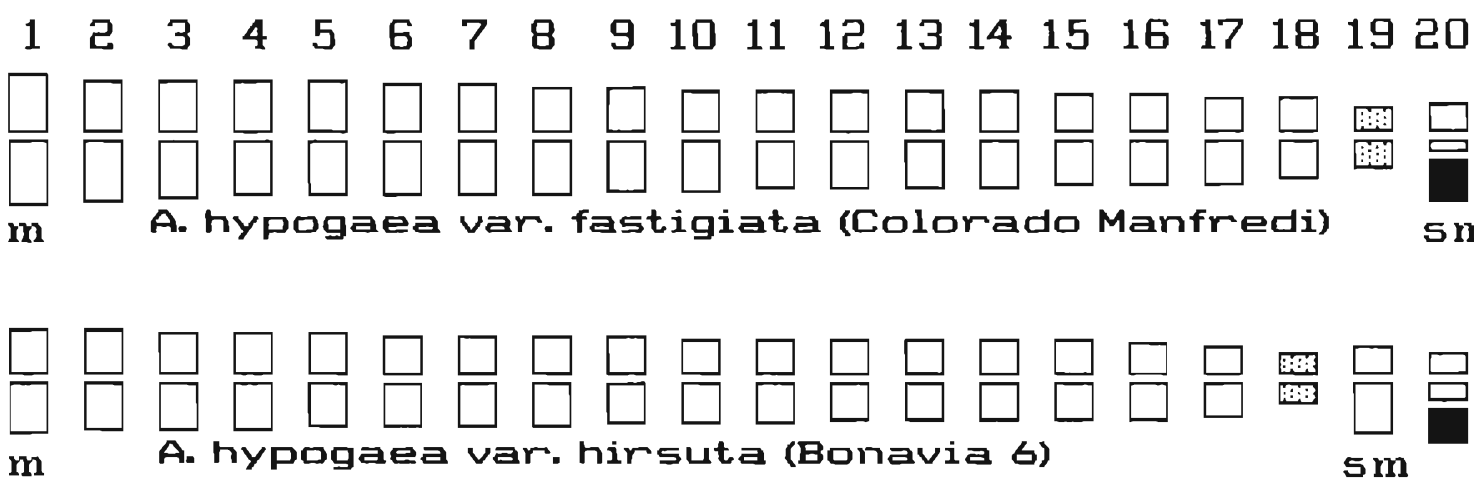

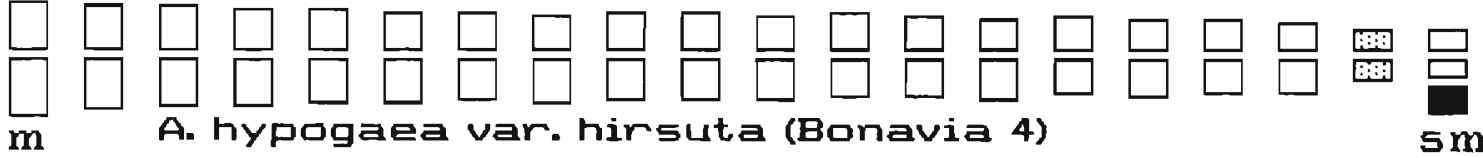

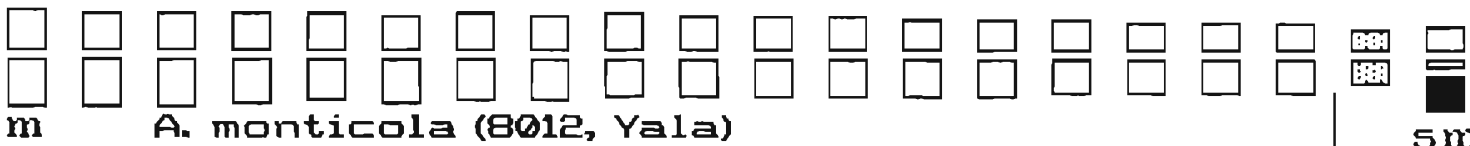

$5 \mathrm{~m}$

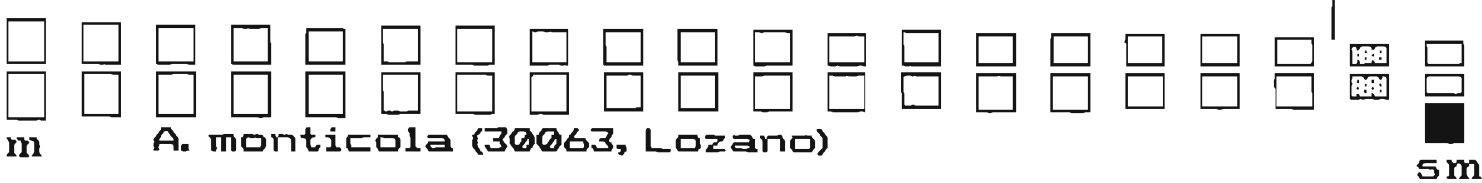

Fig. 8. Cariotipos de especies de Arachis secc. Arachis, tetraploides. Escala igual a $5 \mu \mathrm{m}$. 
7). El par con macrosatélite es del tipo 6 y el par 9 es el "A". El tamaño de los cromosomas varía entre 1.28 y $2.88 \mu \mathrm{m}$. En la accesión 30005 , en una metafase presentaba 8 pares de cromosomas metacéntricos y 2 pares de submetacéntricos, con el cromosoma con macrosatélite del tipo 6.

A. stenosperma, $2 \mathrm{n}=2 \mathrm{x}=20$ (Figs. 15E y 7): tiene los 10 pares de cromosomas metacéntricos. El macrosatélite es del tipo 3, par 9, y el " $\mathrm{A}$ " es el 10. El tamaño de los cromosomas varía entre 1.86 y $4.05 \mu \mathrm{m}$.

A. villosa (357), $2 \mathrm{n}=2 \mathrm{x}=20$ (Figs. $15 \mathrm{~F} y$ 7): posee 8 pares metacéntricos y 2 pares submetacéntricos. El par de cromosomas 10 con satélite es del tipo 3 y el par 8 es el " $\mathrm{A}$ ". El tamaño de los cromosomas varía entre 1.23 y $2.64 \mu \mathrm{m}$.

A. hypogaea subsp. fastigiata var. fastigiata cv. 'Colorado Manfredi' $(2 n=4 x=40)$ : todos los cromosomas son metacéntricos, excepto el par de cromosomas con macrosatélite, que es submetacéntrico (par 20) y es del tipo 3 (Figs. 16A y 8). El par "A" es el 19. El tamaño de los cromosomas varía entre 1.38 y $3.36 \mu \mathrm{m}$.
A. hypogaea ssp. hypogaea var. hypogaea, Nambiquarae $(2 n=4 x=40)$, en la Fig. $15 \mathrm{G}$, se puede observar en profase la coloración diferencial, que sirve para identificar los homólogos (flechas blancas)

A. hypogaea subsp. hypogaea var. hirsuta, Bonavia 6, $2 n=4 x=40$ (Fig. 8): posee un cariotipo diferente del de la primera subespecie. Tiene 18 pares metacéntricos, siendo el par " $A$ " el 18, y dos pares submetacéntricos, uno de los cuales, el par 20, con macrosatéllite del tipo 5. Otra muestra también del Perú, Bonavia 4 (Figs. 16B y 8), posee 19 pares metacéntricos y un par submetacéntrico que corresponde al macrosatélite del tipo 5, siendo el 19 el par " $\mathrm{A}$ ". El tamaño de los cromosomas varía entre 1.18 y $2.89 \mu \mathrm{m}$.

A. monticola, $2 \mathrm{n}=4 \mathrm{x}=40$ : las dos accesiones 8012 de Yala (Fig. 16C) y 30063 de Lozano poseen 19 pares metacéntricos y un par submetacéntrico; este último con macrosatélite del tipo 3 para la primera y del tipo 5 para la segunda, y el par "A" es el 19 en ambas (Fig. 8). El tamaño de los cromosomas varía entre 1.17 y $1.50 \mu \mathrm{m}(8012)$.

Tabla 3. Características cromosómicas de especies del género Arachis, con cariotipos elaborados.

\begin{tabular}{|c|c|c|c|c|c|c|c|}
\hline \multirow[t]{2}{*}{ Especie } & \multirow[t]{2}{*}{ Leg. } & \multirow[t]{2}{*}{$2 n$} & \multirow[t]{2}{*}{$\begin{array}{c}\text { Fórmula del } \\
\text { cariotipo }\end{array}$} & $\begin{array}{c}\text { Cromoma } \\
\text { SAT }\end{array}$ & \multirow{2}{*}{$\begin{array}{c}\text { Comple- } \\
\text { mento: } \\
\text { long. } \\
\text { total } \\
(\mu \mathrm{m})\end{array}$} & \multirow{2}{*}{$\begin{array}{l}\text { Long. } \\
\text { media } \\
\text { de los } \\
\text { crom. } \\
(\mu \mathrm{m})\end{array}$} & \multirow{2}{*}{$\begin{array}{l}\text { Indice } \\
\text { centro- } \\
\text { mérico }\end{array}$} \\
\hline & & & & tipo par & & & \\
\hline
\end{tabular}

\section{Sección Trierectoides}

A. guaranitica

$1056820 \quad 20 \mathrm{~m}$

21

45.48

$2.27 \pm 0.08 \quad 46.63 \pm 0.29$

\section{Sección Erectoides}

$\begin{array}{lrrllllll}\text { A. major } & 555 \mathrm{a} & 20 & 16 \mathrm{~m}+4 \mathrm{sm} & 2 & 2 & 53.54 & 2.76 \pm 0.09 & 43.33 \pm 1.58 \\ \begin{array}{l}\text { A. major } \\ \text { A. major }\end{array} & 14444 & 20 & 20 \mathrm{~m} & 2 & 3 & 61.96 & 3.09 \pm 0.11 & 45.76 \pm 0.46 \\ \begin{array}{l}\text { A. paraquariensis } \\ \text { ssp. capibarensis }\end{array} & 30128 & 20 & 20 \mathrm{~m} & 2 & 3 & 53.78 & 2.69 \pm 0.07 & 46.51 \pm 0.34 \\ \begin{array}{l}\text { A. paraguariensis } \\ \text { ssp. paraguariensis }\end{array} & 573 & 20 & 18 \mathrm{~m}+2 \mathrm{sm} & 3 & 5 & 40.52 & 2.02 \pm 0.06 & 44.12 \pm 1.80 \\ \begin{array}{l}\text { A.paraguariensis } \\ \text { ssp. paraguariensis }\end{array} & 30110 & 20 & 16 \mathrm{~m}+4 \mathrm{sm} & 4 & 7 & 46.92 & 2.34 \pm 0.08 & 42.87 \pm 2.16 \\ \begin{array}{l}\text { A. stenophylla } \\ \text { A. stenophylla }\end{array} & 30115 & 20 & 14 \mathrm{~m}+6 \mathrm{sm} & 4 & 9 & 44.96 & 2.24 \pm 0.08 & 43.55 \pm 2.01 \\ & 572 & 20 & 12 \mathrm{~m}+8 \mathrm{sm} & 4 & 8 & 42.30 & 2.11 \pm 0.06 & 40.01 \pm 2.36 \\ & 30126 & 20 & 14 \mathrm{~m}+6 \mathrm{sm} & 4 & 8 & 43.56 & 2.17 \pm 0.07 & 42.39 \pm 2.44\end{array}$




\section{Sección Caulorrhizae}

A. Pintoi

6791

20
$18 m+2 s m$

$46.74 \quad 2.33 \pm 0.31 \quad 44.04 \pm 1.09$

\section{Sección Procumbentes}

A. Kretschmeri

A. lignosa

$\begin{array}{llllllll}30007 & 20 & 18 m+2 \mathrm{sm} & 9 & 10 & 46.18 & 2.30 \pm 0.10 & 44.62 \pm 1.88 \\ 14248 & 20 & 16 m+4 \mathrm{sm} & 9 & 9 & 46.22 & 2.31 \pm 0.09 & 43.48 \pm 1.68\end{array}$

\section{Sección Arachis}

A. Batizocoi

A. ipaënsis

A. glandulifera

A. magna

A. magna

A. valida

A. valida

A. Cardenasii

A. correntina

A. helodes

A. helodes

A. Kuhlmannii

A. Kuhlmannii

A. Kuhlmannii

A. Kuhlmannii

A. aff. Kuhlmannii

A. duranensis

A. duranensis

A. duranensis

A. duranensis

A. duranensis

A. duranensis

A. duranensis

A. duranensis

A. duranensis

A. Diogoi

A. stenosperma

A. villosa

A. hypogaea ssp.

fastigiata var.

fastigiata Colorado Mdi.

A. hypogaea ssp. hypognea var. hirsuta Bonavia 6

A. hypogaea ssp. hypogaea. var. hirsuta Bonavia 4

A. monticola (Yala)

A. monticola (Lozano)

\begin{tabular}{|c|c|c|c|c|c|c|c|}
\hline 9505 & 20 & $12 m+6 s m+2 s t$ & 5 & 10 & 38.28 & $1.91 \pm 0.05$ & $39.14 \pm 2.63$ \\
\hline 19455 & 20 & $18 m+2 . s m$ & 6 & 5 & 43.46 & $2.17 \pm 0.05$ & $43.87 \pm 1.38$ \\
\hline 30098 & 20 & $8 m+12 s t$ & 3 & 1 & 50.36 & $2.51 \pm 0.10$ & $29.16 \pm 4.53$ \\
\hline 30092 & 20 & $18 \mathrm{ml}+2 \mathrm{sm}$ & 6 & 10 & 40.06 & $2.00 \pm 0.07$ & $44.75 \pm 0.88$ \\
\hline 30097 & 20 & $18 \mathrm{~m}+2 \mathrm{sm}$ & 6 & 10 & 45.36 & $2.26 \pm 0.06$ & $44.68 \pm 0.91$ \\
\hline 30011 & 20 & $20 \mathrm{~m}$ & S & 9 & 45.32 & $2.26 \pm 0.06$ & $46.13 \pm 0.32$ \\
\hline 30147 & 20 & $18 \mathrm{~m}+2 \mathrm{sm}$ & $8-6$ & $7-10$ & 46.76 & $2.33 \pm 0.07$ & $44.31 \pm 1.37$ \\
\hline 36021 & 20 & $16 \mathrm{~m}+4 \mathrm{sm}$ & 5 & 10 & 46.00 & $2.30 \pm 0.12$ & $43.88 \pm 1.82$ \\
\hline 2018 & 20 & $16 \mathrm{~m}+4 \mathrm{sm}$ & 3 & 6 & 52.40 & $2.62 \pm 0.16$ & $42.89 \pm 1.80$ \\
\hline 6325 & 20 & $16 m+4 s m$ & 5 & 10 & 46.08 & $2.30 \pm 0.10$ & $43.05 \pm 1.97$ \\
\hline 6326 & 20 & $16 \mathrm{~m}+4 \mathrm{sm}$ & 5 & 10 & 40.76 & $2.03 \pm 0.10$ & $43.92 \pm 2.01$ \\
\hline 6410 & 20 & $16 n+4 \operatorname{sm}$ & 5 & 10 & 43.30 & $2.16 \pm 0.11$ & $43.66 \pm 1.52$ \\
\hline 6344 & 20 & $16 m+4 \mathrm{sm}$ & 5 & 10 & 46.40 & $2.32 \pm 0.12$ & $43.62 \pm 2.00$ \\
\hline 30017 & 20 & $18 \mathrm{~m}+2 \mathrm{sm}$ & 7 & 10 & 47.14 & $2.35 \pm 0.14$ & $44.05 \pm 1.96$ \\
\hline 6404 & 20 & $16 \mathrm{~m}+4 \mathrm{sm}$ & 5 & 10 & 50.06 & $2.50 \pm 0.14$ & $43.71 \pm 2.48$ \\
\hline 6413 & 20 & $18 \mathrm{~m}+2 \mathrm{sm}$ & 5 & 10 & 52.34 & $2.61 \pm 0.14$ & $44.76 \pm 1.82$ \\
\hline 19539 & 20 & $20 \mathrm{~m}$ & 3 & 7 & 42.06 & $2.10 \pm 0.10$ & $46.09 \pm 0.72$ \\
\hline 19490 & 20 & $18 \mathrm{~m}+2 \mathrm{sm}$ & 3 & 5 & 39.66 & $1.98 \pm 0.11$ & $45.28 \pm 1.37$ \\
\hline 28458 & 20 & $18 \mathrm{~m}+2 \mathrm{sm}$ & 3 & 5 & 34.78 & $1.73 \pm 0.12$ & $44.24 \pm 1.49$ \\
\hline 30061 & 20 & $18 m+2 s m$ & 3 & 3 & 39.70 & $1.98 \pm 0.12$ & $43.99 \pm 1.52$ \\
\hline 30064 & 20 & $18 \mathrm{~m}+2 \mathrm{sm}$ & 3 & 3 & 40.30 & $2.03 \pm 0.13$ & $45.17 \pm 1.06$ \\
\hline 30065 & 20 & $18 m+2 \mathrm{sm}$ & 5 & 10 & 41.16 & $2.05 \pm 0.10$ & $44.07 \pm 2.02$ \\
\hline 30067 & 20 & $18 \mathrm{~m}+2 \mathrm{sm}$ & 5 & 10 & 44.08 & $2.20 \pm 0.13$ & $44.70 \pm 1.92$ \\
\hline 30072 & 20 & $18 \mathrm{~m}+2 \mathrm{sm}$ & 5 & 10 & 40.44 & $2.02 \pm 0.12$ & $44.54 \pm 1.85$ \\
\hline 30078 & 20 & $18 \mathrm{~m}+2 \mathrm{sm}$ & 5 & 10 & 45.44 & $2.27 \pm 0.14$ & $44.06 \pm 1.85$ \\
\hline 6330 & 20 & $18 m+2 s m$ & 6 & 10 & 40.26 & $2.13 \pm 0.14$ & $45.67 \pm 1.30$ \\
\hline 410 & 20 & $20 \mathrm{~m}$ & 3 & 9 & 60.82 & $3.04 \pm 0.19$ & $44.58 \pm 0.87$ \\
\hline 357 & 20 & $16 m+4 s m$ & 3 & 10 & 42.62 & $2.13 \pm 0.12$ & $43.15 \pm 1.72$ \\
\hline 24543 & 40 & $38 m+2 s m$ & 3 & 20 & 103.14 & $2.57 \pm 0.10$ & $45.75 \pm 0.67$ \\
\hline
\end{tabular}

$34261 \quad 40 \quad 36 \mathrm{~m}+4 \mathrm{sm} \quad 5 \quad 20$

$85.52 \quad 2.13 \pm 0.07 \quad 45.02 \pm 1.05$

$34260 \quad 40 \quad 38 m+2 \mathrm{sm} \quad 5 \quad 20$

$85.56 \quad 2.13 \pm 0.08 \quad 45.26 \pm 0.85$

$801240 \quad 38 \mathrm{ml}+2 \mathrm{sm}$

320

$80.72 \quad 2.01 \pm 0.06 \quad 45.32 \pm 0.48$ $3006340 \quad 38 \mathrm{~m}+2 \mathrm{sm}$

520

$80.74 \quad 2.01 \pm 0.06 \quad 45.53 \pm 0.93$ 


\section{Discusión}

En las especies donde se pudo establecer el cariotipo de varias accesiones, algunas presentaron el mismo cariotipo, como en $A$. helodes y A. magna; sin embargo, en otras especies hubo diferencias notables, como en $A$. major, accesión $555 a$, con respecto a las accesiones 14444 y 30128. En $A$. paraguariensis se analizaron tres accesiones; dos pertenecientes a la ssp. paraguariensis y una a la ssp. capibarensis; las tres presentaron cariotipos diferentes. También hay diferencias, en algunos casos muy notables en los cariotipos de $A$. duranensis, $A$. Kuhlmannii, A. valida, A. stenophylla, A. hypogaea y A. monticola.

Stalker \& Dalmacio (1981), Singh \& Moss (1982) y Cai et al. (1987) publicaron los cariotipos de algunas especies de la sección Arachis. A fin de comparar los resultados, se tomaron los datos publicados por los mencionados autores, se determinó la razón del BL/ $\mathrm{BC}$, con lo que se estableció la morfología de los cromosomas de acuerdo con la clasificación de Levan et al. (1964): metacéntrico (m) $\mathrm{BL} / \mathrm{BC}=1$ a 1,7 ; submetaméntrico $(\mathrm{sm}) \mathrm{BL} /$ $\mathrm{BC}=1,7$ a 3 . Con estos datos se confeccionó la tabla 4. A. stenosperma es la única especie en la que coinciden los autores de tres de los trabajos, respecto de la presencia de 20 metacéntricos; Cai et al . (1987), por el contrario determinaron $14 \mathrm{~m}+4 \mathrm{sm}+2$ st. En A. villosa, A. correntina y A. Cardenasii coinciden Stalker y Singh, pero difieren Cai et al. y los presentes autores. Para A. Batizocoi se determinaron cariotipos diferentes en los cuatro trabajos.

Stalker (1991) estudió 4 accesiones en $A$. glandulifera, encontrando tres de ellas (30098, 30099 y 30100$)$ con igual cariotipo, con $14 \mathrm{~m}+4$ $s m+2 s t$, y una accesión (30091) con $10 m+8$ $\mathrm{sm}+2$ st. En el presente trabajo se estudió la accesión 30098 (Fig. 13C), estableciéndose un cariotipo compuesto por $8 m+12$ st, y sólo un par de cromosomas con satélite (Fig. 4). Stalker menciona tres pares con satélite para las tres accesiones de igual cariotipo y dos pares para

Tabla 4. Comparación de los cariotipos observados por diversos autores.

\begin{tabular}{|c|c|c|c|c|}
\hline Especie & $\begin{array}{c}\text { Stalker \& } \\
\text { Dalmacio (1981) }\end{array}$ & $\begin{array}{c}\text { Singh \& Moss } \\
\quad(1982)\end{array}$ & $\begin{array}{l}\text { Cai et al. } \\
\text { (1987) }\end{array}$ & $\begin{array}{l}\text { presentes } \\
\text { autores }\end{array}$ \\
\hline A. villosa & $18 m+2 s m$ & $18 m+2 s m$ & $16 m+4 s m$ & $16 m+4 s m$ \\
\hline A. correntina & $18 \mathrm{~m}+2 \mathrm{sm}$ & $18 m+2 s m$ & $16 m+4 s m$ & $16 m+4 s m$ \\
\hline A. stenosperma & $20 \mathrm{~m}$ & $20 \mathrm{~m}$ & $14 m+4 s m+2 s t$ & $20 \mathrm{~m}$ \\
\hline A. Cardenasii & $20 \mathrm{~m}$ & $20 \mathrm{~m}$ & $16 m+4 s m$ & $16 \mathrm{~m}+4 \mathrm{sm}$ \\
\hline A. Batizocoi & $20 \mathrm{~m}$ & $18 m+2 s m$ & $16 \mathrm{~m}+4 \mathrm{sm}$ & $12 m+6 s m+2 s t$ \\
\hline A. Diogoi (1) & $20 \mathrm{~m}$ & $20 \mathrm{~m}$ & & $18 m+2 s m$ \\
\hline A. duranensis (2) & $18 m+2 s m$ & $20 \mathrm{~m}$ & & $18 \mathrm{~m}+2 \mathrm{sm}$ \\
\hline A. duranensis (3) & $20 \mathrm{sm}$ & $18 m+2 s m$ & & $18 m+2 \mathrm{sm}$ \\
\hline A. Rigonii & & & $12 m+6 s m+2 t$ & $16 \mathrm{~m}+4 \mathrm{sm}$ \\
\hline A. monticola & & $40 \mathrm{~m}$ & $32 m+8 s m$ & $38 m+2 s m$ \\
\hline \multicolumn{5}{|l|}{ A. hypogaea } \\
\hline ssp. hypogaea & & $40 \mathrm{~m}$ & $32 m+8 s m$ & $38 m+2 s m$ \\
\hline \multicolumn{5}{|l|}{ A. hypogaea } \\
\hline ssp. fastigiata & & $40 \mathrm{~m}$ & $32 m+8 s m$ & $38 \mathrm{~m}+2 \mathrm{sm}$ \\
\hline
\end{tabular}

(1) En Stalker \& Dalmacio (1981) y Singh \& Moss (1982), como A. chacoensis (GK 10602).

(2) K 7988, Salta, Campo Durán, igual que KMoF 19490.

(3) En Stalker \& Daimacio (1981), como A. spegazzinii (GKP 10038) y en Singh \& Moss (1982) como A.sp. (GKP 10038), Salta, El Prado, igual localidad que KScGl 28458. 
la accesión 30091. Probablemente, lo que Stalker menciona como cromosomas con satélite sean los cromosomas subtelocéntricos que, a primera vista, semejan cromosomas con satélite, pero que serían pseudosatélites (Battaglia, 1955). El par 1 del cariotipo publicado por Stalker sería el par con constricción secundaria. Probablemente, la diferencia en los resultados de los dos trabajos se deba a que nosotros pretratamos el material durante 3 horas y Stalker sólo durante 15 minutos.

La simetría del cariotipo da una idea de la evolución, la tendencia general es que las especies con cariotipos más simétricos son más primitivas (Stebbins, 1971). La información obtenida en este trabajo sobre la evolución de las especies de Arachis mediante el análisis de los cariotipos está en correlación con el resultado del estudio de la exomorfología (Krapovickas, 1973). A. guaranitica y A. tuberosa de la sección Trierectoides, serían las especies más primitivas por su exomorfología. Una de estas especies, $A$. guaranitica, posee el cariotipo más simétrico, con 10 pares metacéntricos, y el índice centromérico con el promedio más elevado.

La especie más primitiva de la sección Erectoides a juzgar por la simetría del cariotipo sería A. major (14444 y 30128), con los 10 pares metacéntricos. La más evolucionada sería $A$. stenophylla, por poseer el cariotipo más asimétrico: $12 \mathrm{~m}+8 \mathrm{sm}$. Es interesante señalar aquí que en esta especie, el par de cromosomas con satélite es del tipo 4 , mientras que en la más primitiva es del tipo 2 (Tabla 1 ).

Dentro de la sección Arachis, interpretada como la más evolucionada, hay tres especies: A. stenosperma, A. duranensis (Sc.19539) y $A$. valida (30011), que tienen 10 pares metacéntricos. Estas especies son las que presentan el cariotipo más simétrico, por lo que serían las más primitivas de la sección. A. valida se diferencia de las otras dos por carecer del cromosoma " $\mathrm{A}$ " y por el par de cromosomas con satélite que es del tipo 8 , mientras que en A. stenosperma y $A$. duranensis es del tipo 3. Las especies con cromosomas "A" y con el cariotipo más asimétrico $(16 \mathrm{~m}+4 \mathrm{sm})$ son $A$. villosa, $A$. helodes, A. Cardenasii y A. correntina. La especie sin el par "A" y de cariotipo más asimétrico es $A$. glandulifera, con $8 \mathrm{~m}+12 \mathrm{st}$, siendo también la especie con el índice centromérico más bajo. En segundo lugar estaría A. Batizocoi, con $12 \mathrm{~m}+6 \mathrm{sm}+2 \mathrm{st} / \mathrm{sm}$.

En el maní cultivado, A. hypogaea, Krapovickas \& Rigoni (1960) y Krapovickas y Gregory (1994) reconocen dos subespecies: hypogaea y fastigiata. La primera tiene el par de cromosomas con satélite del tipo 5, mientras que la segunda tiene el tipo 3 (Tabla 3). Es interesante señalar aquí que Husted (1936) ya había determinado $38 \mathrm{~m}+2 \mathrm{sm}$; también había indicado que el par de cromosomas con satélite era submetacéntrico en $A$. hypogaea, "bunch type" (ssp. fastigiata) y "runner type" (ssp. hypogaea). Si bien no diferenció los dos tipos de cromosomas con satélite en las dos subespecies, de acuerdo con las figuras 2 (ssp. fastigiata) y 3 (ssp. hypogaea) que ilustran su trabajo, los cromosomas con satélite corresponderían a los tipos designados aquí como 3 y 5 respectivamente.

La longitud total del complemento en la ssp. hypogaea var. hirsuta es de 85,52 $\mu \mathrm{m}$, mientras que en la ssp. fastigiata var. fastigiata es de 103,14 $\mu \mathrm{m}$. Resslar et al. (1981), al medir el contenido de ADN en la ssp. hypogaea var. hypogaea, encontraron 10,4 pg, en la ssp. fastigiata var. fastigiata $11,1 \mathrm{pg}$ y en la ssp. fastigiata var. vulgaris $11,3 \mathrm{pg}$. El mayor tamaño de los cromosomas en la ssp. fastigiata corresponde al mayor contenido de ADN, es decir, el tamaño de los cromosomas sería directamente proporcional al contenido de ADN.

A. monticola, $2 n=4 x=40$, tiene relación con el origen del maní (Krapovickas \& Rigoni, 1957), pues es la única especie que produce híbridos fértiles al cruzarla con $A$. hypogaea. En dos accesiones obtenidas en la Prov. de Jujuy, en Yala y Lozano, localidades que distan unos 5 a $6 \mathrm{~km}$ una de otra, los cromosomas SAT son diferentes: en Yala del tipo 3 y en Lozano del tipo 5. El material de Yala tiene el par de cromosomas SAT similar al de A. hypogaea ssp. fastigiata; en cambio, el de Lozano tiene el par SAT similar al de la ssp. hypogaea

Singh \& Moss (1982) encontraron en $A$. monticola dos pares de cromosomas con satélite; sin embargo, nosotros hemos observado un solo par de cromosomas con satélite en tres procedencias. Probablemente, lo señalado como satélite en la fotografía publicada por 
Singh \& Moss (1982) sería más bien una condensación diferencial en la región subdistal del brazo.

Las especies que poseen cromosomas con satélite del tipo 9 pertenecen a la sección Procumbentes, sin considerar $A$. benensis de la sección Arachis. Satélites del tipo 4 solamente se han encontrado en especies de la sección Erectoides, mientras que el tipo 3 se halla en las secciones Erectoides, Extranervosae, Caulorrhizae, Rhizomatosae y Arachis . Cromosomas con satélite del tipo 2 se encuentran en especies de las secciones Trierectoides, Erectoides, Caulorrhizae y Extraneroosae. Cromosomas con satélite del tipo 1 se encuentra en una sola accesión de $A$. villosulicarpa de la sección Extraneroosae. Los tipos 5, 6,7 y 8 se

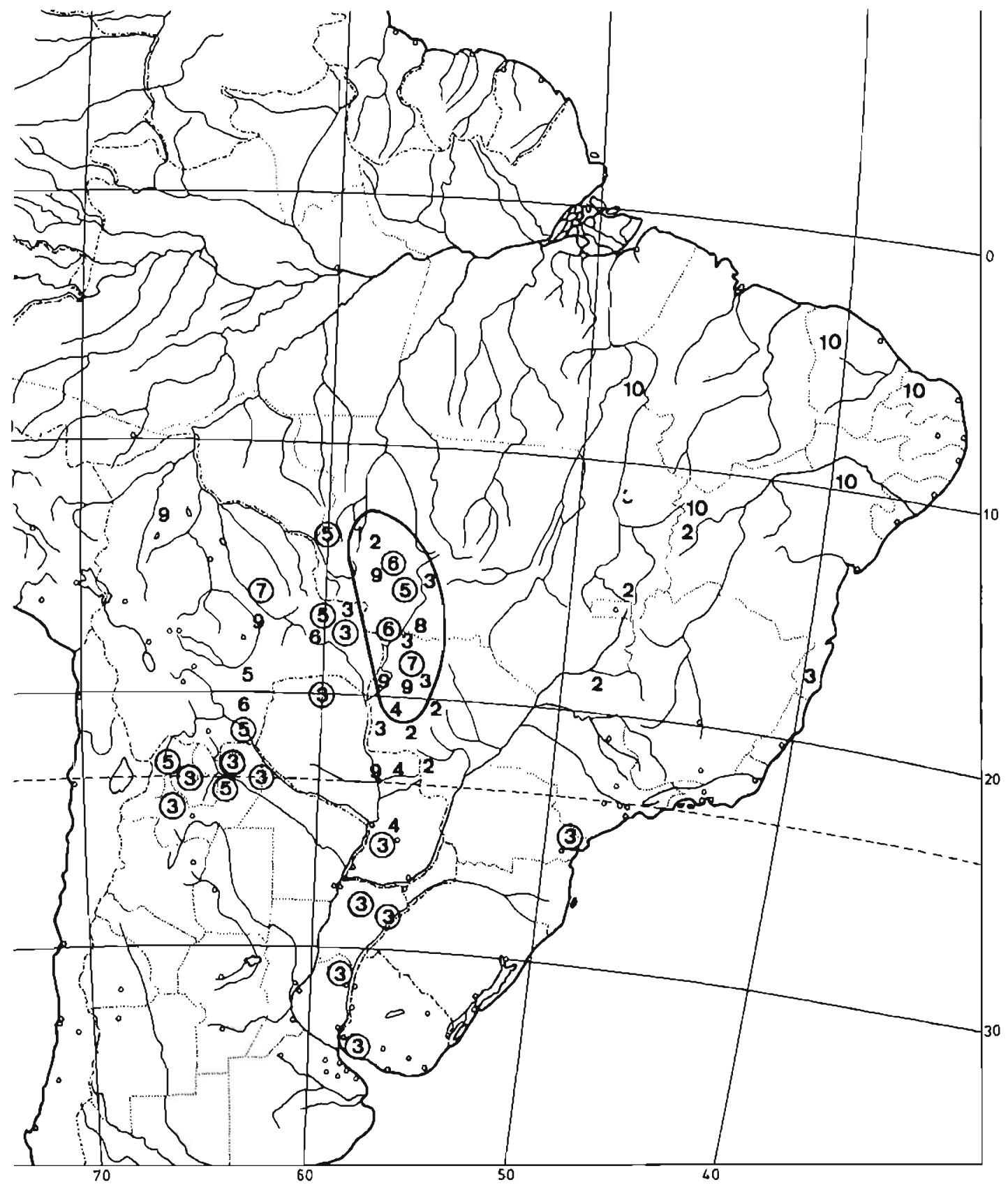

Fig. 9. Distribución geugráfica de los cromusumas "A" (0) y SAT (tipos 1 a 10). 
encuentran exclusivamente en la sección Arachis. Es decir, que en la sección Arachis se encuentra mayor cantidad de especies con diferentes tipos de cromosomas con satélite, lo que indicaría que esta sección está en pleno proceso de evolución cariotípica.

El mapa (Fig. 9) muestra el centro de variación (Mato Grosso) de los distintos tipos de cromosomas con satélite, que coincide perfectamente con el centro de variación morfológica y química. Krapovickas (1973) considera al meridiano $57^{\circ}$ como el eje del área de distribución del género Arachis, encontrándose al W de este meridiano la mayoría de las especies con el par " $\mathrm{A}$ ". Otro hecho interesante de destacar es que al Este de dicho meridiano se encuentran las especies que poseen cromosomas con satélite del tipo 2, probablemente el más primitivo entre los diez tipos mencionados.

En el cuadro de la Fig. 10 se ordenaron los 10 tipos de cromosomas SAT, agrupando a la izquierda los que tienen el segmento proximal pequeño y a la derecha los que tienen el segmento proximal de tamaño similar al del brazo 1 . Se señala también la presencia del cromosoma " $A$ ", por lo cual un cromosoma SAT tipo 3 fue desplazado hacia la derecha. En la línea basal se señalan los niveles de ploidía hallados para cada tipo de cromosoma SAT, solo o asociado con el cromosoma " $\mathrm{A}$ ". Las especies poliploides presentan solamente los cromosomas SAT tipo 3 y 5.

El ordenamiento resultante sugiere relaciones entre los diferentes cromosomas SAT, las que se indican con flechas. EL tipo 10, hallado en $A$. sylvestris, figura aislado por no ser clara su vinculación con el resto.

En cada caso se indican sólo las secciones involucradas, ya que la lista completa de especies ordenadas por tipo de cromosoma se encuentra en la Tabla 3.

A la izquierda aparecen todas las secciones del género y a la derecha y hacia arriba únicamente la sección Arachis.

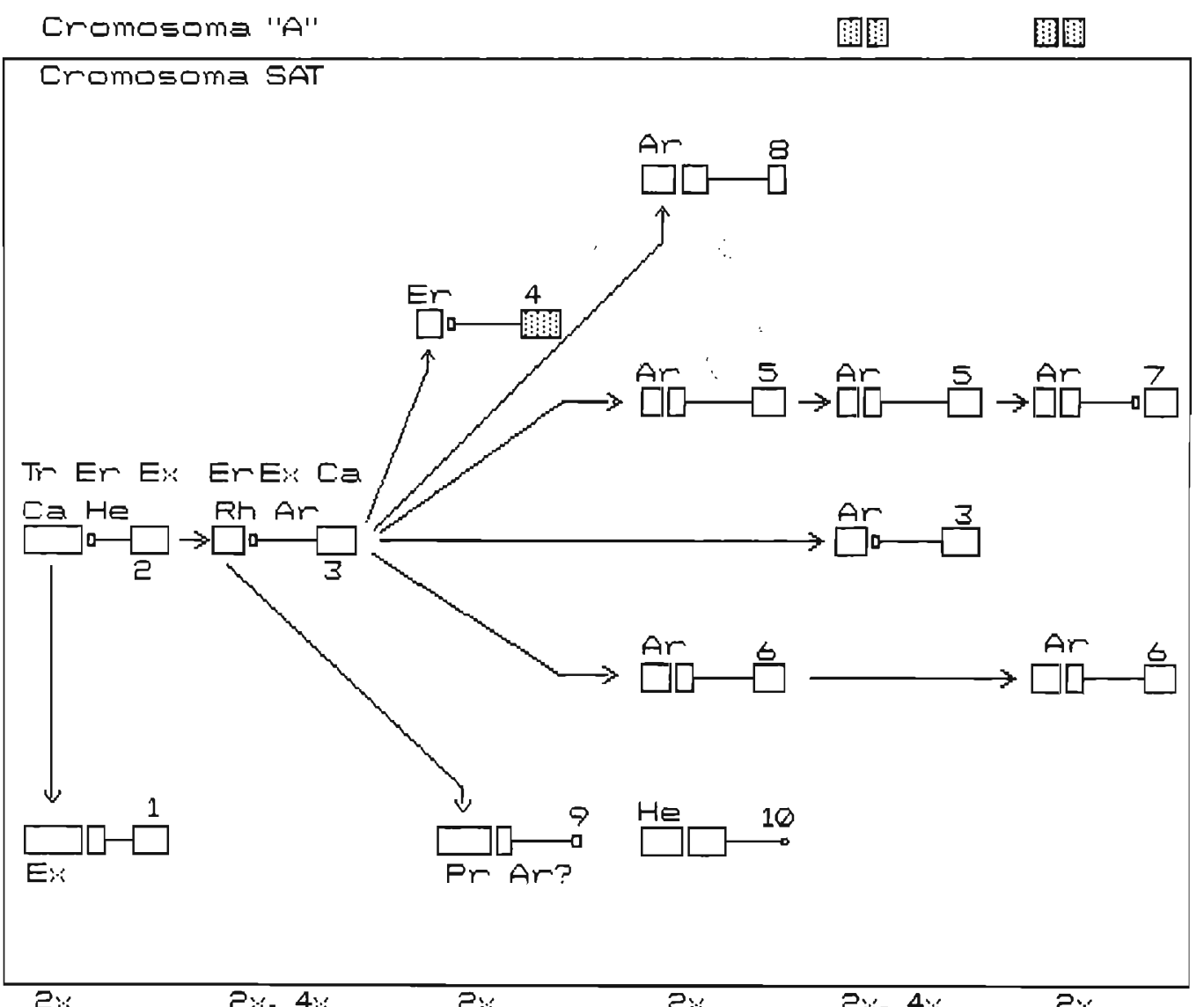

Fitg. 11. Probable evolucion estructural del cromosoma SAT. 
A los cromosomas tipo 2 y 3 corresponde la mayor variabilidad exomorfológica, ya que se encuentran en casi todas las secciones del género (7), excepto en Procumbentes, que posee el tipo 9 .

Los cruzamientos realizados por M.P. Gregory \& W.C. Gregory (1979) y tratados por Krapovickas \& Gregory (1994) involucran solamente especies con cromosomas SAT de los tipos 2,3 y 9 .

La sección Arachis presenta una variabilidad notable del cromosoma SAT (tipos 3, 5, 6, 7,8 y 9); el cromosoma " $\mathrm{A}$ " aparece únicamente en la sección Arachis, asociado con cromosomas SAT de los tipos 3, 5, 6 y 7 .

Es posible que los cromosomas SAT tipo 2 y 3 sean los más primitivos del género por estar presentes en la mayor cantidad de secciones y también en la mayor cantidad de especies del género.

También es posible que la variabilidad del par de cromosoma SAT y la aparición del par "A" en la sección Arachis sean posteriores, como se indica con la dirección de las flechas.

Es probable que la variabilidad del cromosoma SAT se deba tanto a inversiones como a translocaciones, como se deduce de la variación de longitud total y longitud relativa de sus partes.

Determinar el origen genético del maní cultivado es importante, tanto desde el punto de vista teórico como práctico. El maní cultivado es un alotetraploide segmentario (Stebbins, 1957), posee un par de cromosomas "A" y un par de cromosomas SAT (Fig. 16A, B y C). Gregory \& Gregory (1976 y 1979) sugieren que A. hypogaea se habría formado por la hibridación entre especies de la sección Arachis. Uno de los progenitores diploides $(2 n=2 x=20)$ tendría que llevar cromosomas "A" y el otro carecer de ellos. Smartt et al. (1978) propusieron a A. Cardenasii, con cromosomas "A" (genoma " $\mathrm{A}$ ") y a $A$. Batizocoi, sin ellos (genoma " $B$ "), como los probables progenitores diploides de $A$. hypogaea. De acuerdo con los resultados presentados en este trabajo, consideramos que $A$. duranensis y $A$. ipaënsis serían las especies progenitoras más probables del maní cultivado. Se llega a esta conclu- sión por el cariotipo, la morfología y la distribución geográfica. Cuando Smartt et al. (1978) propusieron su teoría, se conocía únicamente una sola especie, $A$. Batizocoi, sin el par "A"; actualmente se conocen otras 6 especies: A. ipaënsis, A.glandulifera, A. valida, A. magna, A. Hoehnei y A. benensis.

Por el cariotipo pueden descartase fácilmente A. Batizocoi, A. glandulifera y $A$. valida. Las otras dos especies son parecidas entre si, pero $A$. ipaënsis es la que presenta mayor similitud con $A$. hypogaea.

Por otro lado, por el análisis de los RFLP (Kochert et al., 1991, Paik-Ro et al., 1992) y de las isozimas (Lu \& Pickersgill, 1993) se descarta a A. Batizocoi como probable progenitor diploide del maní cultivado. Para los últimos autores, $A$. glandulifera sería, por las isozimas, el progenitor diploide sin el par " $A$ ", pero los datos citológicos aportados por Stalker (1991) y por los presentes autores, lo descartan como antecesor del maní cultivado. Sobre la base de los RFLP, Kochert et al. (1991) proponen a A. ipaënsis y A. duranensis como los probables progenitores diploides de $A$. hypogaea; por otro lado, también Paik-Ro et al. (1992) proponen a A. duranensis como progenitor del maní cultivado.

Los progenitores diploides del maní cultivado propuestos tienen un par de cromosomas con constricción secundaria, mientras que $A$. hypogaea posee un sólo par de cromosomas con satélite. Esto probablemente se deba a que exista anfiplastia, es decir, un par de cromosomas con satélite inhibe la manifestación del otro par. Este efecto ha sido observado en otros géneros y muy bien estudiado en Triticum y Aegilops (Lacadena et al. 1988).

\section{Agradecimientos}

Este trabajo fue llevado a cabo mediante el apoyo económico de las siguientes instituciones: CAFPTA, CONICET, FAO y Secretaría de Ciencia y Técnica (UNNE). También deseamos expresar nuestro agradecimiento a los Dres. C. Simpson, J.F.M. Valls y al Agr. J.R. Pietrarelli, quienes gentilmente nos han cedido semillas de algunas especies de maní. 


\section{Bibliografía}

BATTAGLIA, E. 1955. Chromosome morphology and terminology. Caryologia 8(1): 179-187.

CAI, Q., S. Lu \& C.C. CHINNAPPA. 1987. Analysis of karyotypes and Giemsa C-banding patterns in eight species of Arachis. Genome 29: 187-194.

FERNANDEZ, A. 1973. El ácido láctico como fijador cromosómico. Bol. Soc. Argent. Bot. 15:287-290.

GREGORY, M. P. et W. C. GREGORY. 1979. Exotic germ plasm of Arachis L. interspecific hybrids. J. Hered. 70: 185-193.

GREGORY, W.C. 1946. Peanut breeding program underway. Res. and Farming 5. Prog. Rep. 4: 41-45.

GREGORY, W.C. \& M.P. GREGORY. 1976. Groundnut, pp. 151-154. In N.W. Simmonds (Ed.), Evolution of crop plants. Longman Group, Ltd., London.

HUNZIKER, J.H. 1961. Estudios cromosómicos en Cupressus y Libocedrus (Cupressaceac). Rev. Invest. Agric. 15(2): 169-185.

HUSTED, L. 1933. Cytological studies of the peanut Arachis. 1. Chromosome number and morphology. Cytologia 5: $109-117$.

1936. Cytological studies of the peanut Arachis. II. Chromosome number, morphology and behaviour, and their application to the problem of the origin of the cultivated forms. Cytologia 7: 396-423.

KOCHERT, G., T. HALWARD, W.D. BRANCH \& C.E. SIMPSON. 1991. RFLP variability in peanut (Arachis hypogaea L.) cultivars and wild species. Theor. Appl. Genet. 81: 565-570.

KRAPOVICKAS, A. 1973. Evolution of the genus Arachis, pp. 135-151. In R. Moav (Ed.). Agricultural genetics Selected topics. Wiley. New York.

KRAPOVICKAS, A., A. FERNANDEZ\& P.SEELIGMANN. 1974. Recuperación de la fertilidad en un híbrido interespecífico estéril de Arachis (Leguminosue). Bonplandia 3: 129-142.

KRAPOVICKAS;, A. \& W.C. GREGORY. 1994. Revisión del género Arachis (Leguminosae). Bonplandia 8: $1-186$.

KRAPOVICKAS, A. \& V.A. RIGONI. 1951. Estudios citológicos en el género Arachis. Rev. Invest. Agric. 5(3): 289-293.

1957. Nuevas especies de Arachis vinculadas al problema del origen del maní. Darwiniana 11(3): 431-458. 1960. La nomenclatura de las subespecies y variedades de Arachis hypogaea L. Rev. Invest. Agric. 14(2): 197-228.

KURABAYASHI, M., H. LEWIS \& P. H. RAVEN. 1962. A comparative study of mitosis in the Onagraceae. Amer. J. Bot. 49: 1003-1026.

LACADENA, J.R., M.C. CERMEÑO, J. ORELLANA \& J.L. SANTOS. 1988. Nucleolar competition in Triticeae. In Brandham, P.E. ed. Kew Chromosome Conference III. 151-165. London.

LEVAN, A., K. FREDGA \& A. A. SANDBERG. 1964. Nomenclature for centromeric position on chromosomes. Hereditas 52: 201-220.
LIMA-De-FARIA, A. 1956. The role of the kinetochore in chromosome organization. Hereditas 42: 85-160.

1980. Classification of genes, rearrangements and chromosomes according to the field. Hereditas 93:146.

LU, J. \& B. PICKERSGILL. 1993. Isozyme variation and species relationships in peanut and its wild relatives (Arachis L. - Leguminosae). Theor. Appl. Genet. 85: 550 560.

PAIK-RO, O.G., R.L. SMITH \& D.A. KNAUFT. 1992. Restriction fragment length polymorphism evaluation of six peanut species within Arachis section. Theor. Appl. Genet. 84: 201-208.

RAMAN, V.S. 1981. Nature of chromosome pairing in allopolyploids of Arachis and their stability. Cytologia 46: 307-321.

RAMAN, V.S. \& P.C. KESAVAN. 1965. A tetraploid hybrid in Arachis and its bearing on differentiation between diploid species. Indian J. Genet. PI. Breed. 25: $80-84$.

RESSLAR, P.M. \& W.C. GREGORY. 1979. A cytological study of three diploid species of the genus Arachis L. J. Hered. 70: 13-16.

RESSLAR, P.M., J.M. STUCKY \& J.P. MIKSCHE. 1981. Cytophotometric determination of the amount of DNA in Arachis L. Sect. Arachis (Leguminosae). Amer. J. Bot. 68(2): 149-153.

SINGH, A.K. 1986. Utilization of wild relatives in the genetic improvement of Arachis hypogaea L. 8. Synthetic amphidiploids and their importance in interspecific breeding. Theor. Appl. Genet. 72: 433439.

SINGH, A.K. \& J.P. MOSS. 1982. Utilization of wild relatives in genetic improvement of Arachis hypogaea L. Part 2: Chromosome complements of species in section Arachis. Theor. Appl. Genet. 61: 305-314.

SMARTT, J. 1964. Cross-compatibility relationships between the cultivated peanut Arachis hypogaca L. and other species of the genus Arachis. PhD thesis, North Carolina State University at Raleigh.

SMARTT, J., W.C. GREGORY \& M.P. GREGORY. 1978. The genomes of Arachis hypogaea. I. Cytogenetic studies of putative genome donors. Euphytica 27: 665-675.

STALKER, H.T. 1991. A new species in section Arachis of peanuts with a D genome. Amer. J. Bot. 78(5): 630637.

STALKER, H.T. \& R.D. DALMACIO. 1981. Chromosomes of Arachis species, section Arachis. J. Hered. 72: 403408.

STALKER, H.T., J.S. DHESI \& D.C. PARRY. 1991a. An analysis of the genome species Arachis batizocoi (Fabaceae). Pl. Syst. Evol. 174: 159-169.

,,$---\&$ J.H. Hahn. 1991b. Cytological and interfertility relationships of Arachis section Arachis. Amer. J. Bot. 78(2): 238-246.

STEBBINS, G.L. 1957. Genetics, evolution and plant breeding. Indian J. Genet. Pl. Breed. 17: 129-141.

- 1971. Chromosomal evolution in higher plants. Edward Arnold Publ. Ltd., London. 

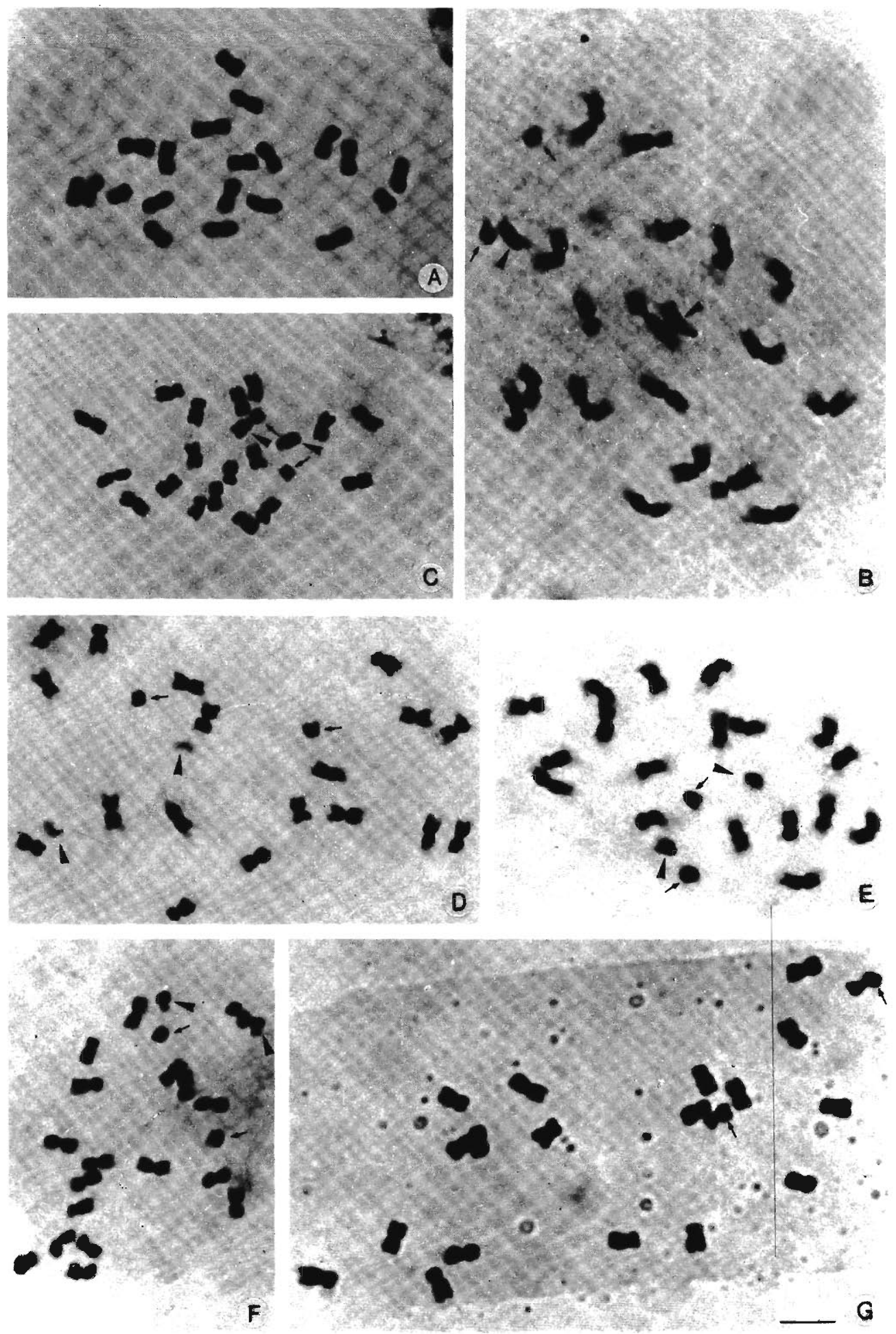

Fig. 11. Cromosomas somáticos de Arachıs, $2 \mathrm{n}=20$. A. guaranitica (10582): A, metafase. A. major (555a): B, protase; C, metafase. A. paraguariensis ssp. paraguariensis (30124): D, profase. A. paraguariensis ssp. capibarensis (573): E, profase; F, metafase. A. stenophylla (572): G, metafase. Escala igual a $5 \mu \mathrm{m}$. Cromosomas SAT: $\Delta$ brazo $1+$ segmento proximal, $\uparrow$ satélite. 


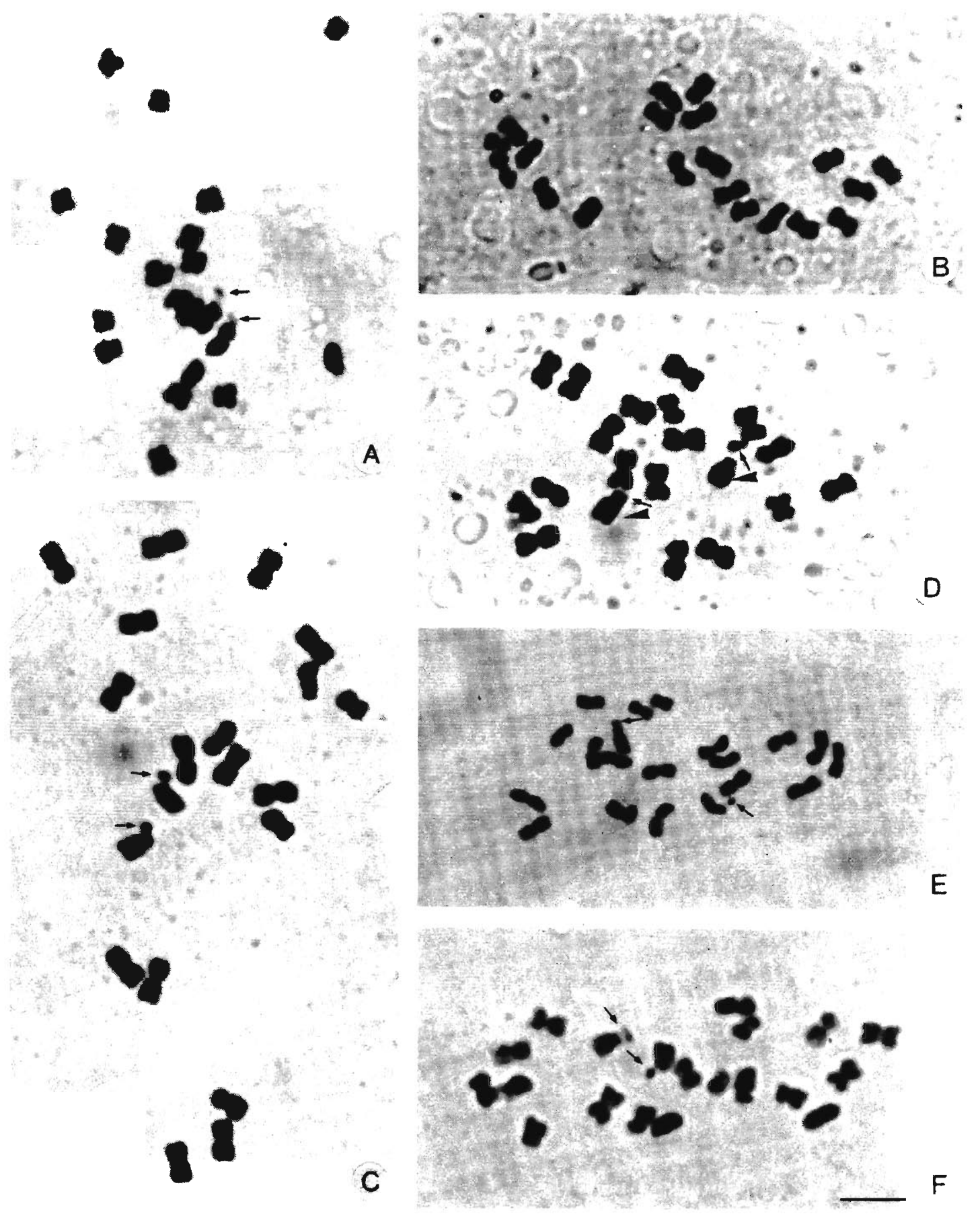

Fig. 12. Cromosomas somáticos de Arachis, $2 \mathrm{n}=20$. A. sylvestris (10891): A, metafase. A. Pintoi (12787): $\mathrm{B}$, metafase. A. lignosa (14248): C, metafase. A. Rigonii (10034): D, metafase. A. Kretschmeri (30007): E, metafase. A. appressipila (34574): F, metafase. Escala igual a $5 \mu \mathrm{m}$. Cromosomas SAT: $\Delta$ brazo $1+$ segmento proximal, $\uparrow$ satélite. 

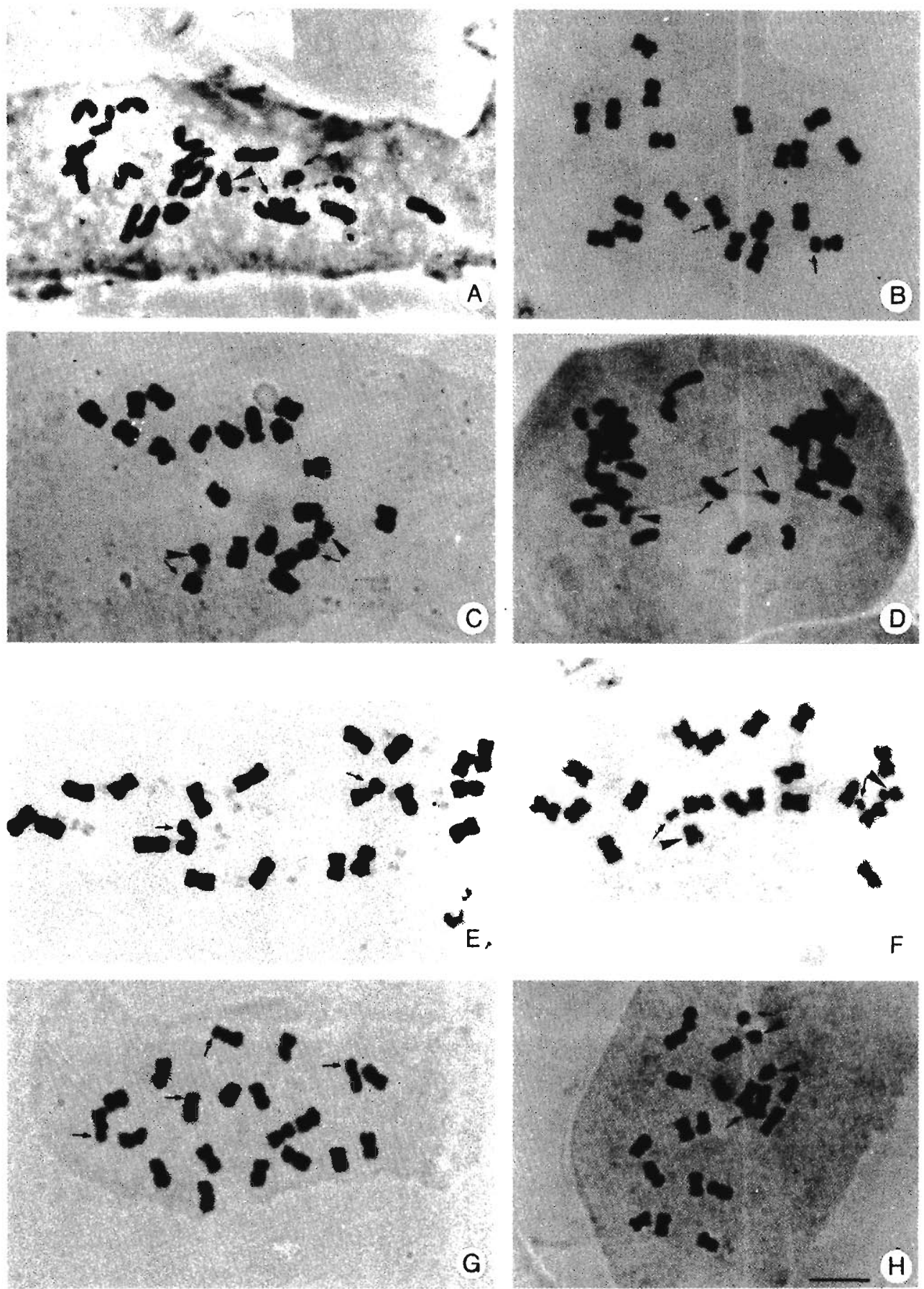

Fig. 13. Cromosomas somáticos de Arachis, 2n=20. A. Batizocoi (9505): A, metafase sin pretratamiento. A. ipaënsis (19455): B, metafase. A. glandulifera (30098): C, metafase; D, anafase. A. magna (30092): E, metafase. A. valida (30011): F, metafase. A. valida (30147): G, metafase. A. aff. prostrata (5913): H, metafase. Escala igual a $5 \mu$ m. Cromosomas SAT: $\Delta$ brazo $1+$ segmento proximal, $\uparrow$ satélite. 

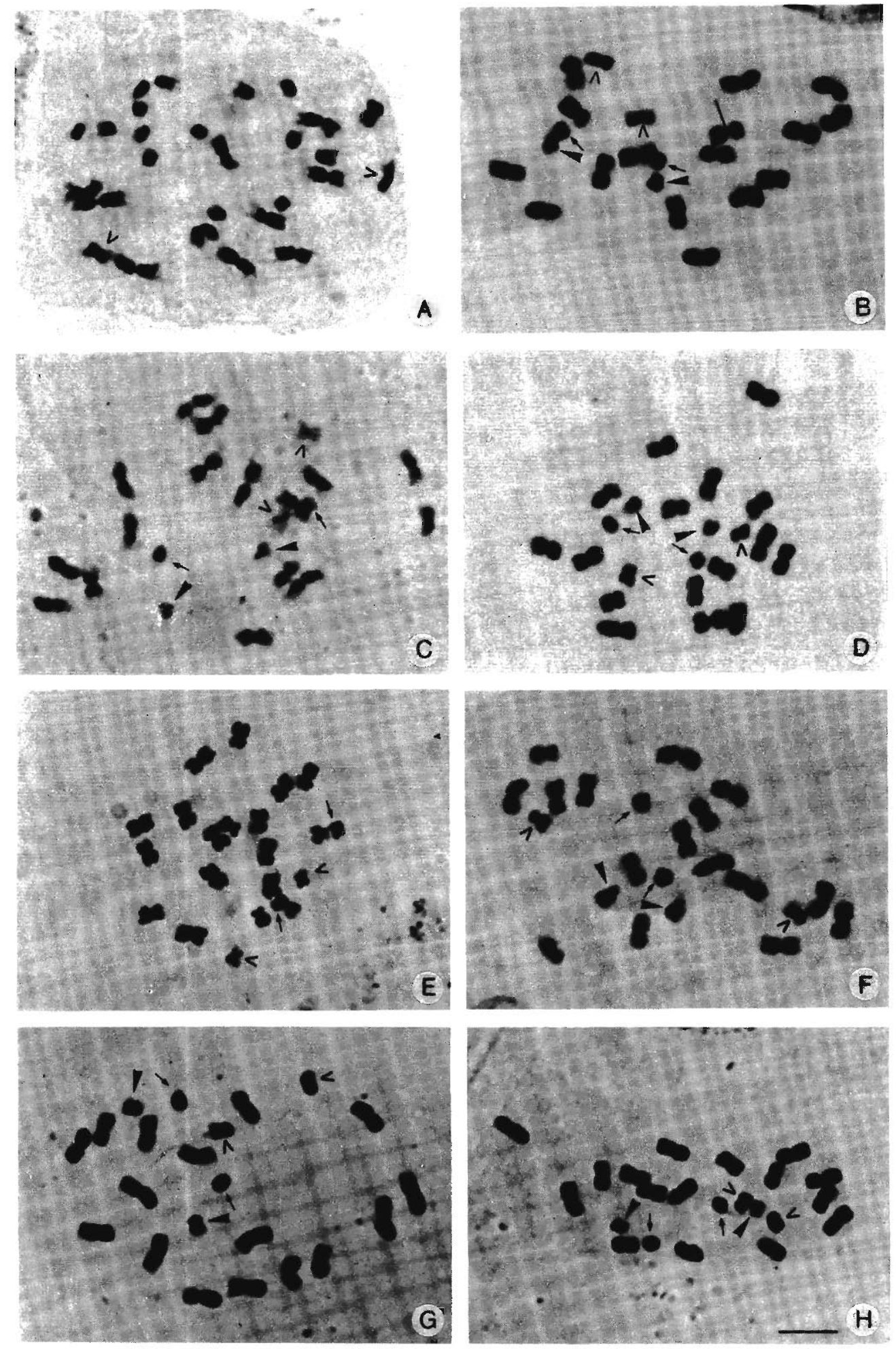

Fig. 14. Cromosomas somáticos de Arachis, $2 \mathrm{n}=20$. A. Cardenasii (36015): A, profase; B, metafase. A. correntina (2018): C, profase. A. duranensis, metafases: D, 19490. E, 30061. F, 30064. G, 30072. H, 30078. Escala igual a 5 um. Cromosomas SAT: $\Delta$ brazo $1+$ segmento proximal, $\uparrow$ satélite. Cromosomas "A": ^. Centrómero extendido: I. 

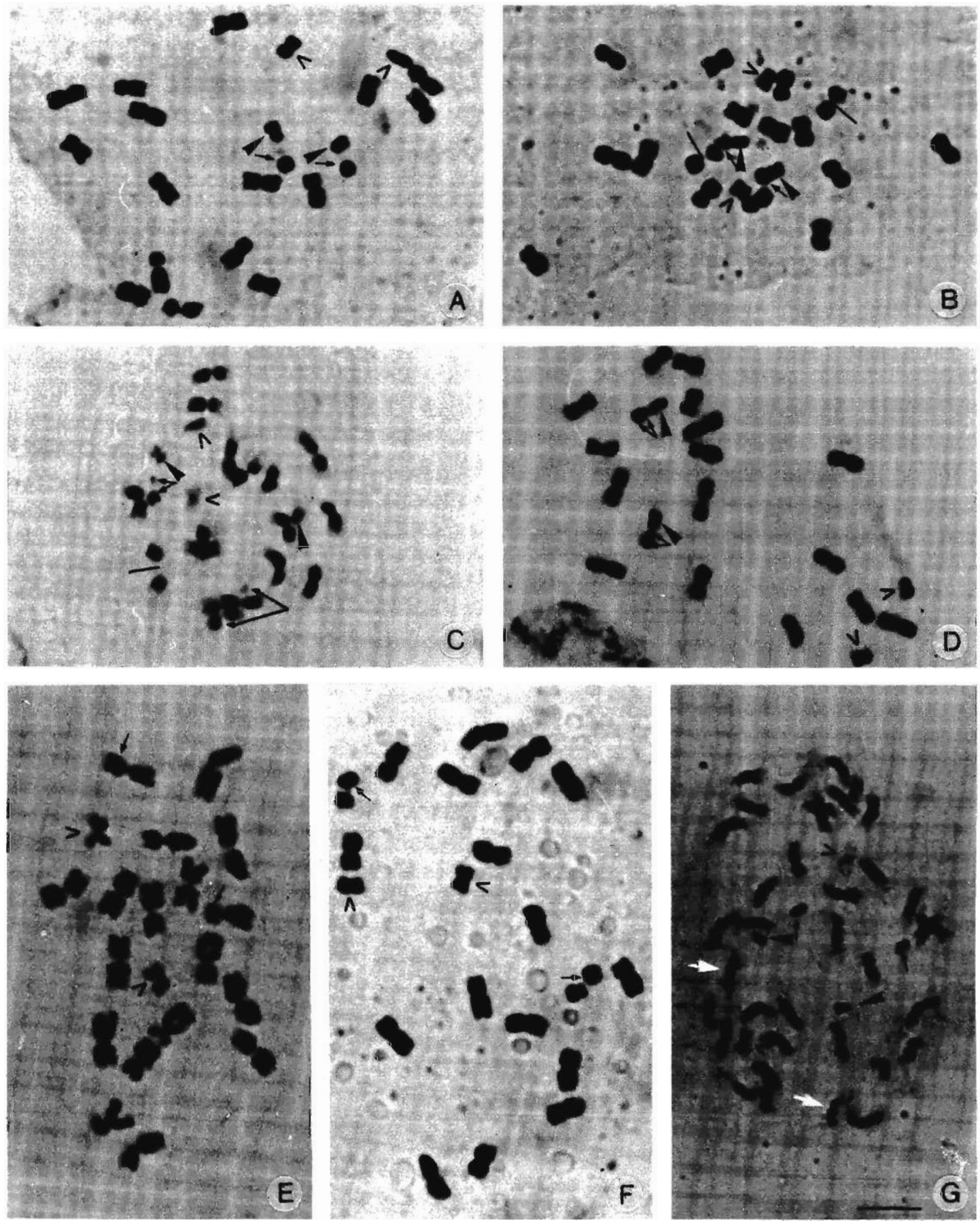

Fig. 15. Cromosomas somáticos de Arachis. A. helodes (6325) $2 \mathrm{n}=20$ : A, metafase. A. Kuhlmannii, 2n=20: B, 6344, metafase; C y D, 30017, profase y metafase respectivamente. A. stenosperma (410), 2n=20: E, metafase. A. villosa (357), 2n=20: $\mathbf{F}$, metafase. A. hypogaea ssp. hypogaea var. hypogaea (Nambiquarae), $2 \mathrm{n}=40: \mathrm{G}$, profase, $2 \mathrm{n}=40$. Escala igual a $5 \mu \mathrm{m}$. Cromosomas SAT: $\boldsymbol{\Delta}$ brazo $1+$ segmento proximal, $\uparrow$ satélite. Cromosomas " $\mathrm{A}$ ": $\wedge$. Centrómero extendido: I. Flechas blancas: coloración diferencial. 

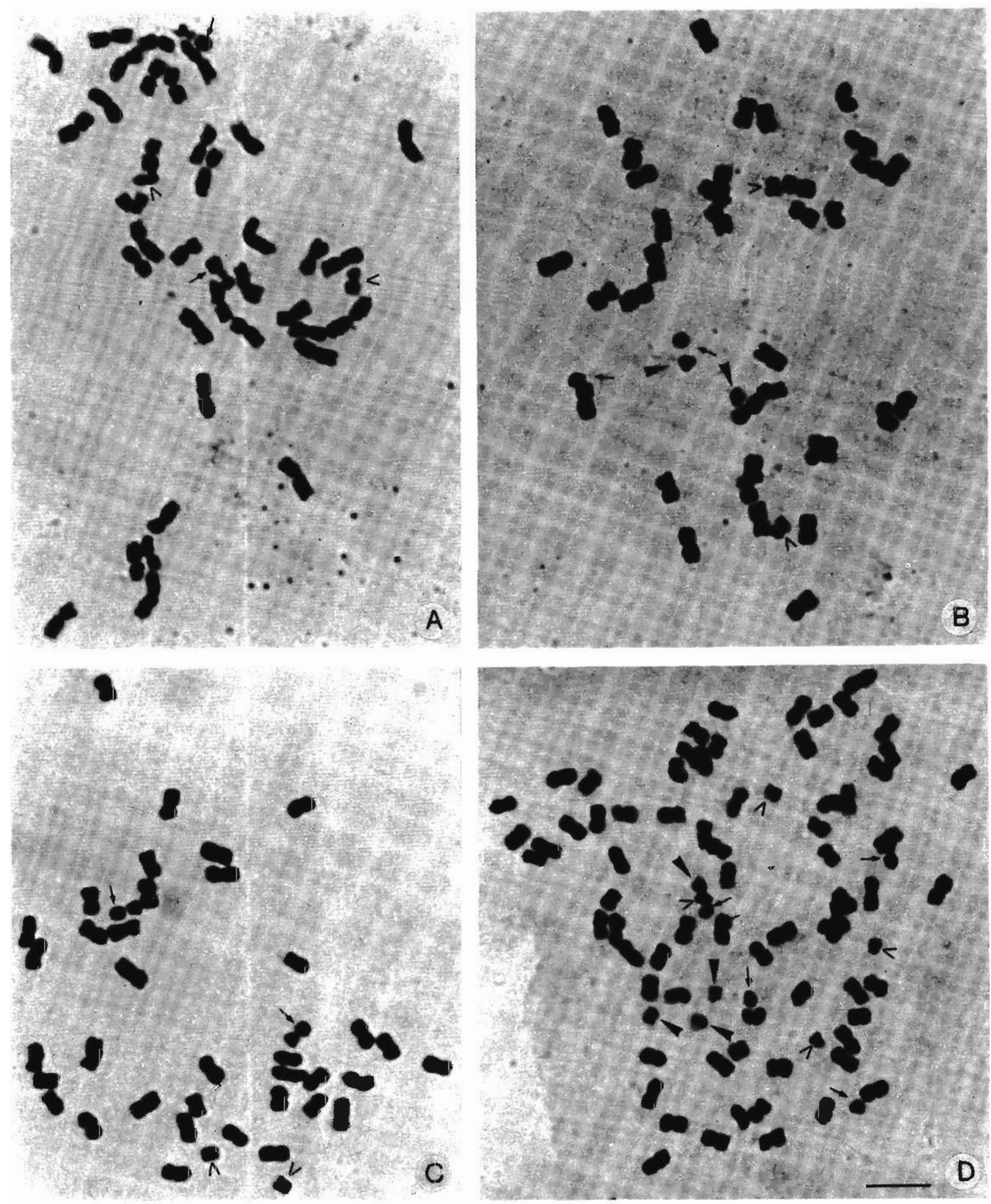

Fig. 16. Cromosomas somáticos de Arachis. A. hypogaea ssp. fastigiata var. fastigiata cv. 'Colorado Manfredi', $2 \mathrm{n}=40: \mathbf{A}$. metafase. A. hypogaea ssp. hypogaea var. hypogaea (Bonavia 4), $2 \mathrm{n}=40$ : B, metafase. A. monticola: C, 8012, metafase, $2 \mathrm{n}=40$; $\mathrm{D}, 30062$, célula polisomática, $2 \mathrm{n}=80$. Escala igual a $5 \mu \mathrm{m}$. Cromosomas SAT: $\mathbf{\Delta}$ brazo $1+$ segmento proximal, $\uparrow$ satélite. Cromosomas "A": $\wedge$. 